\title{
Interaction between a surface quasi-geostrophic buoyancy filament and an internal vortex
}

\author{
JEAN N. REINAUD $\dagger^{*}$, DAVID G. DRITSCHEL $\dagger$ and XAVIER CARTON $\ddagger$ \\ † University of St Andrews, Mathematical Institute, North Haugh, St Andrews, KY16 9SS, UK \\ ‡ Laboratoire d'Océanographie Physique et Spatiale, IUEM, UBO/UBL, 29280 Plouzané, France \\ (18 August 2016)
}

\begin{abstract}
This paper focuses on the nonlinear interaction between a surface quasi-geostrophic buoyancy filament and an internal vortex. We first revisit the stability of an isolated buoyancy filament. The buoyancy profile considered is continuous and leads to a continuous velocity field, albeit one with infinite shear just outside its edge. The stability properties of an isolated filament help to interpret the unsteady interaction with a sub-surface (internal) vortex studied next. We find that, in all cases, the filament breaks into billows, analogous in form to those occurring in Kelvin-Helmholtz shear instability. For intense buoyancy filaments, the vortex itself may undergo strong deformations, including being split into several pieces. Generally, the nonlinear interaction causes both the filament and the vortex to lose their respective 'self'-energies to the energy of interaction. The flow evolution depends sensitively on whether the vertical vorticity of the filament and the vortex have the same or opposite signs - termed "cooperative" and "adverse" shear respectively. In cooperative shear, the filament rolls up into a coherent surface eddy above a vortex initially placed below it, whereas in adverse shear, buoyancy is expelled above the vortex. Although sufficiently great shear induced by the buoyancy filament may split the vortex in both cases, adverse shear is significantly more destructive.
\end{abstract}

Keywords: Vortex dynamics; Semi-quasigeostrophy; Quasigeostrophy

\section{Introduction}

Modern satellite (infra red) imagery and sea surface altimetry are providing an increasingly sharp picture of the complex nature of the dynamics of the oceans, a complexity which has been underestimated for decades. These data primarily allow one to visualise and measure the dynamical activity of the oceans at their surface, and here buoyancy filaments are common and conspicuous features, see for example Gula, Molemaker and McWilliams (2014), McWilliams et al. (2015), Iermano et al. (2012) and references therein. These data (in particular sea-surface temperature and sea-surface height) have also been used in attempts to reconstruct the threedimensional structure of oceanic mesoscale currents (Isern-Fontanet et al. 2008, Wang et al. 2013) and generally to infer sub-surface ocean dynamics (Ciani et al. 2015). However, it is clear that the surface of the oceans does not entirely control ocean dynamics at depth. It is now well documented that the ocean interior is populated by a large number of eddies or vortices. Examples include mediterranean water eddies observed in the Atlantic (Meddies). It is estimated that between 35 and 40 Meddies may be found in the North Atlantic at all times, see Richardson, McCartney and Maillard (1991), Richardson, Bower, Zenk (2000), and Barbosa Aguiar, Peliz and Carton (2013), Sokolovskiy, Filuyshkin and Carton (2013). Furthermore, Filyushkin et al. (2010) estimate that 150 to 200 vortex lenses populate an area of approximately $1,800 \mathrm{~km}$ in radius centred on the Portimão Canyon.

While such vortices may have a surface signature (e.g. an elevated or depressed sea-surface

The University of St Andrews is a charity registered in Scotland No SC013532

${ }^{*}$ Corresponding author: Email: jean.reinaud@st-andrews.ac.uk 
height), not all sub-surface vortices do, and moreover it can be difficult to disentangle several vortices. In any case, a surface signature alone does not provide sufficient information to deduce the depth, size and intensity of a vortex independently.

In this paper, we study the interaction between an active ocean surface and an active interior in an idealised, yet archetypical situation. We examine, theoretically and computationally, the interaction between a filament of surface buoyancy and an internal vortex under the quasigeostrophic approximation. The buoyancy profile is chosen to be a continuous distribution to avoid the unphysical feature of a singular velocity field at its edges. The 'elliptical' distribution chosen has the unique property of having uniform shear within the filament, and also permits a semi-analytical treatment of its linear stability. The filament idealises the surface filaments commonly observed in the oceans, and its instabilities are well known to generate small scales. For the interior, we chose a simple, spherical vortex consisting of uniform potential vorticity (in coordinates stretched vertically by the ratio of the buoyancy to Coriolis frequencies). This model set-up is arguably the simplest one for studying the effects of buoyancy filaments on sub-surface vortices, and vice versa. Simulations show surprising rich and complex dynamics, depending on parameters like the filament-vortex intensity ratio, their vertical and horizontal offset, etc.

The paper is organised as follows. $\S 2$ describes the mathematical formulation of the model. $\S 3$ revisits the linear stability of a buoyancy filament and illustrates its nonlinear evolution. $\S 4$ addresses the interaction between the filament and an internal vortex. The influence of the geometry of the interaction as well as the relative intensities of the vortex and the filament are investigated. Here we also examine the energy transfers which take place, showing that generally the vortex and filament self-energies are depleted and transferred into interaction energy. A few conclusions are drawn in $\S 5$.

\section{Formulation}

In order to explore parameter space widely, we simplify the problem to a single surface buoyancy filament having an 'elliptical' profile (see below) interacting with a sub-surface vortex of uniform potential vorticity (PV). Moreover, we employ the quasi-geostrophic (QG) approximation, which filters inertia-gravity waves and reduces the dynamics to material conservation of PV and surface buoyancy. Nonetheless, material advection is nonlinear, and the interior PV and surface buoyancy interact nontrivially via a nonlocal, three-dimensional flow field.

The QG approximation is widely used in studies of geophysical fluid dynamics. It is strictly valid for $\mathrm{Fr}^{2} \ll R o \ll 1$ where $\mathrm{Fr}=U /(N H)$ is the Froude number and $R o=U /(f L)$ is the Rossby number, see e.g. Vallis (2006) for details of the derivation. Here, $U$ is a characteristic (horizontal) flow speed, $L$ and $H$ are characteristic horizontal and vertical length scales, while $f$ and $N$ are the basic-state Coriolis and buoyancy frequencies, both taken constant in this study. In fact, the QG approximation continues to be accurate for moderate Froude and Rossby numbers, so long as they are less than unity (Tsang and Dritschel, 2015), even over time scales long compared to $(R o f)^{-1}$.

The mean surface of the ocean is located at $z=0$. We consider first an ocean of infinite depth in $\S 3$ to describe, in the simplest manner, the linear and nonlinear dynamics of the buoyancy filament in the absence of an internal vortex. For the nonlinear simulations of the filament-vortex interaction in $\S 4$, a finite-depth ocean is considered for practical reasons. Here, we describe the quasi-geostrophic model in the simpler situation of infinite depth.

The layerwise two-dimensional incompressible fluid flow derives from a scalar streamfunction $\psi$ which can be written as the sum of the streamfunction $\psi_{i}$ induced by the interior distribution of potential vorticity anomaly $q$, and the streamfunction $\psi_{s}$ induced by the surface buoyancy 
$b$,

$$
\psi=\psi_{i}+\psi_{s},
$$

with, for the part due to the interior,

$$
\Delta \psi_{i}=q,
$$

where $\Delta=\partial^{2} / \partial x^{2}+\partial^{2} / \partial y^{2}+\partial^{2} / \partial z^{2}$ is the three-dimensional Laplacian. In $\Delta$, the vertical coordinate $z$ has been stretched by the frequency ratio $N / f$. In this way, neither $f$ nor $N$ appear in the re-scaled equations of motion. The streamfunction $\psi_{s}$ associated with the surface buoyancy is a harmonic function

$$
\Delta \psi_{s}=0,
$$

with

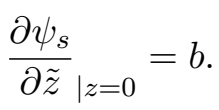

Here, $b=-g \rho^{\prime} /\left(\rho_{0} N\right)$ is the buoyancy scaled by $N$ ( $b$ then has units of velocity). The final equations express material conservation of both $q$ and $b$ in the absence of forcing, viscous and diabatic effects,

$$
\frac{D q}{D t}=0 \quad \text { and } \quad \frac{D b}{D t}=0
$$

where $D / D t=\partial / \partial t+u \partial / \partial x+v \partial / \partial y$ and where the velocity components $u=-\partial \psi / \partial y$ and $v=\partial \psi / \partial x$ are evaluated at the same depth as the advected variable.

\section{Stability of the surface buoyancy filament}

We start by revisiting the stability of a filament of surface buoyancy in the absence of an internal vortex. This is done using the standard SQG model where the fluid domain is semi-infinite (and defined here for $z \geq 0$ ). Formal aspects of the dynamics of surface quasi-geostrophy (SQG) are described in Held et al. (1995). It should be noted that Lapeyre and Klein (2006) indicate that SQG dynamics itself provides a good approximation of the surface dynamics even beyond the formal validity of quasi-geostrophy theory. Juckes (1995) analysed the linear stability of a filament of uniform buoyancy. The discontinuity of the buoyancy distribution at the edge of the filament is associated with a logarithmic singularity of the velocity field along the edge. In the same paper, the author extended his results to estimate the growth rates for a filament with smoothed edges to remove the velocity singularity. We revisit the stability problem using a different buoyancy distribution which corresponds to a simpler (linear) velocity field within the filament, with no singularity at its edge. This buoyancy distribution stems from an exact solution for elliptical distributions of surface buoyancy obtained in Dritschel (2011). These solutions are obtained from the limiting forms of three-dimensional ellipsoidal volumes of uniform potential vorticity (cf. Dritschel, Reinaud and McKiver (2004)) for which the vertical axis length is shrunk to zero. By infinitely stretching one of the axes of the ellipse, one obtains a filament of buoyancy which exhibits the same structure for the internal velocity distribution, namely a linear dependence on the cross-filament coordinate. This distribution was used by Scott (2011) to study the nonlinear evolution of a buoyancy filament. Here, we address its linear stability.

Consider a filament of width of $2 a$ lying parallel to the $x$-axis and centred on $y=0$. The 

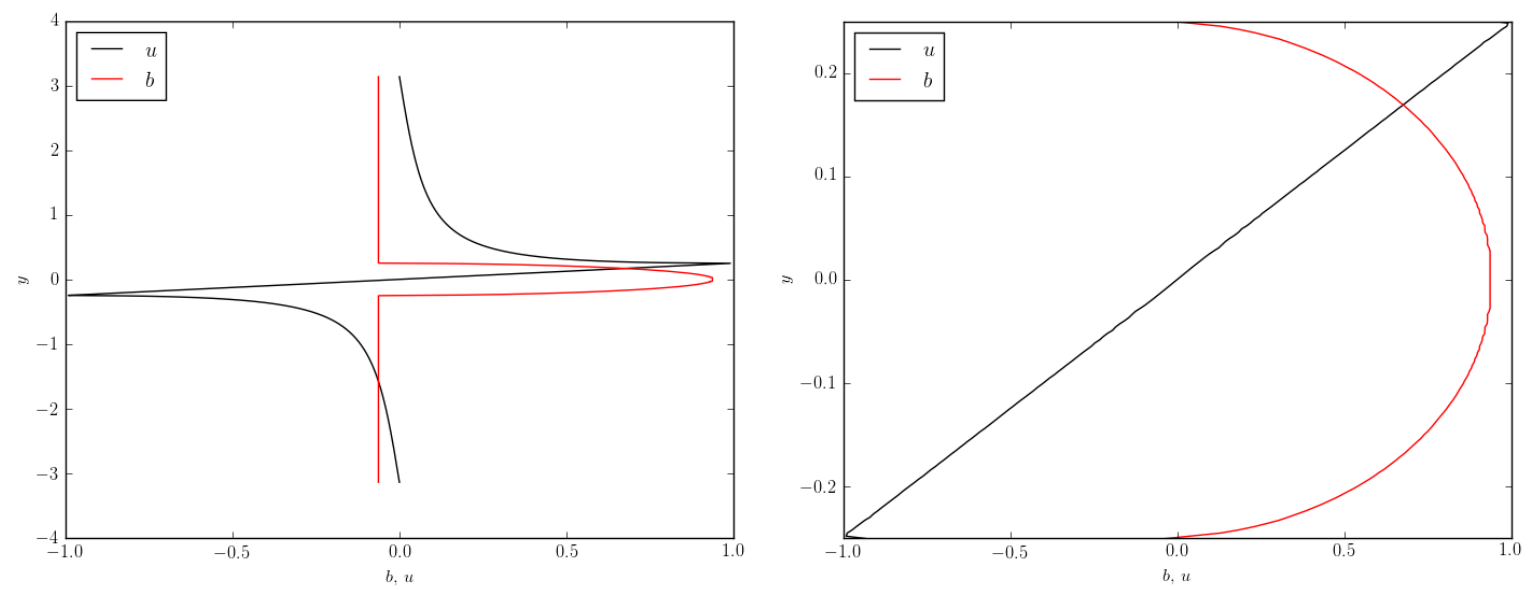

Figure 1. Left: surface buoyancy profile $b$ (red) and corresponding along-filament velocity profile $u$ (black). The velocity is obtained numerically from the code used for the nonlinear simulations. The image on the right provides a close-up of the internal region where velocity field is linear.

buoyancy distribution in the $y$-direction (cross-section) is given by

$$
\bar{b}(y)= \begin{cases}b_{m} \sqrt{1-\left(\frac{y}{a}\right)^{2}}, & \text { for }-a<y<a \\ 0, & \text { for }|y| \geq 0\end{cases}
$$

in equilibrium. Because of its shape and origin, this profile will henceforth be referred to as the 'elliptical' profile, following Scott (2011). The maximum buoyancy of the filament is $b_{m}$. This buoyancy profile induces a linear, along-filament velocity field inside the filament,

$$
u=U_{e}(y) \equiv \frac{b_{m}}{a} y \quad \text { for } \quad-a<y<a .
$$

In equilibrium the cross-filament velocity $v$ is zero by symmetry. The basic-state configuration is illustrated in figure 1.

Consider a straight material line $y=\bar{y}$ of constant buoyancy in equilibrium. We displace the material line by a small perturbation $\eta(x, \bar{y}, t)$ in the $y$-direction, without changing the buoyancy attached to the fluid particles. Thus, the displaced material line is $y=\bar{y}+\eta(x, \bar{y}, t)$. As it retains its original buoyancy, we have $b(y)=\bar{b}(\bar{y})=\bar{b}(y-\eta)$. Linearising this, we obtain

$$
b(y)=\bar{b}(y)-\eta \bar{b}_{y}, \text { where } \bar{b}_{y} \equiv \frac{d \bar{b}}{d y} .
$$

Here and below, linearisation allows us to replace $\bar{y}$ by $y$ in $\eta(x, \bar{y}, t)$. Hence, the (Eulerian) buoyancy perturbation is given by

$$
b^{\prime}(x, y, t) \equiv b(y)-\bar{b}(y)=-\eta(x, y, t) \bar{b}_{y}(y) .
$$

The evolution of the displacement $\eta$ is obtained from the linearised kinematic condition

$$
\frac{\partial \eta}{\partial t}+U_{e}(y) \frac{\partial \eta}{\partial x}=v(x, y, t)
$$

where $v$ is the linearised cross-filament velocity due to the perturbation. As the basic flow is independent of $x$, we can consider displacements in the form of a monochromatic wave $\eta(x, y, t)=\check{\eta}(y) e^{\mathrm{i}(k x-\sigma t)}$ with wavenumber $k$. Substituting this in the equation above and evaluating $v$ (see Appendix 1), we obtain

$$
\sigma \check{\eta}(\theta)=U_{e} k \check{\eta}(\theta)+b_{m} \frac{k}{\pi} \int_{0}^{\pi} \check{\eta}\left(\theta^{\prime}\right) \cos \theta^{\prime} K_{0}\left(k a\left(\cos \theta-\cos \theta^{\prime}\right)\right) d \theta^{\prime},
$$



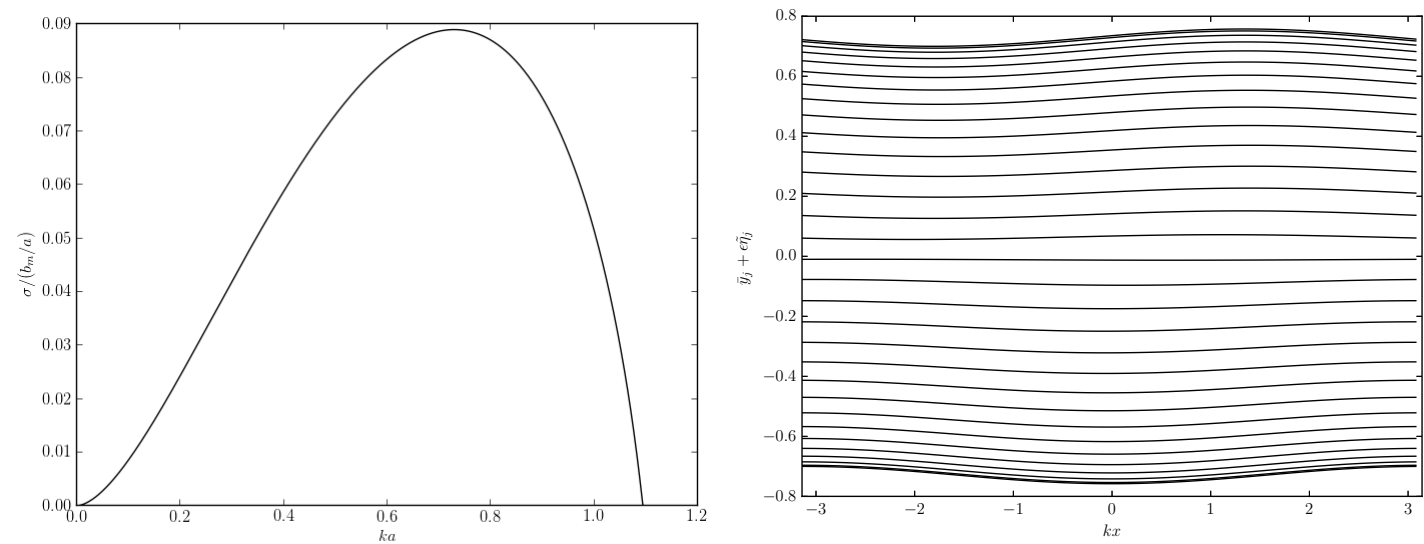

Figure 2. Linear stability of the buoyancy filament with an elliptical profile. Left: non-dimensional growth rate $\sigma a / b_{m}$ vs the non-dimensional wavenumber $k a$. Right, illustration of the eigenmode for the most unstable mode with $k a \simeq 0.729$ and $\sigma a / b_{m} \simeq 0.089$.

where we have used the coordinate transformation $y(\theta)=-a \cos \theta, 0<\theta<\pi$. The integral on the right-hand side is calculated numerically by discretising $\theta$ into $N$ equally spaced intervals of width $\Delta \theta=\pi / N$. Over each interval, we take the displacement amplitude to be a constant denoted by $\check{\eta}_{m}$ for $1 \leq m \leq N$. This results in the algebraic system,

$$
\frac{\sigma a}{b_{m}} \check{\eta}_{j}=k a\left(-\check{\eta}_{j} \cos \theta_{j}+\sum_{m=1}^{N} \check{\eta}_{m} G_{j, m}\right), \quad 1 \leq j \leq N,
$$

where $G_{j, m}$ is the kernel

$$
\frac{1}{\pi} \int_{\theta_{m}-\Delta \theta / 2}^{\theta_{m}+\Delta \theta / 2} \cos \theta^{\prime} K_{0}\left(k a\left(\cos \theta_{j}-\cos \theta^{\prime}\right)\right) d \theta^{\prime}
$$

obtained by two-point Gaussian quadrature over each discrete layer $m$. This is an $N \times N$ matrix eigenvalue problem for $N$ eigenvalues $\sigma=\sigma_{j}(1 \leq j \leq N)$ and $N$ corresponding eigenvectors $\eta_{j, m}(1 \leq j, m \leq N)$ giving the spatial structure of the perturbation. Instability occurs if the imaginary part of any eigenvalue is positive.

Results are presented in figure 2. Here, we have used $N=2000$. The uniform shear $b_{m} / a$ inside the filament is used to normalise the growth rates $\sigma$. The longitudinal wavenumber $k$ is normalised by $a^{-1}$, where $a$ is the filament's half thickness. The left panel shows the maximum normalised growth rate, while the right panel shows the structure of the corresponding eigenmode in terms of the displaced buoyancy contours,

$$
y_{m}(x)=\bar{y}_{m}+\epsilon \Re\left(\eta_{m} e^{\mathrm{i} k x}\right)
$$

where $\Re$ denotes the real part, $\bar{y}_{m}$ is the equilibrium centerline location of the $m^{\text {th }}$ discrete layer, and $\epsilon$ is an arbitrary amplitude used for illustration purposes. The lines of constant phase tilt to the left with increasing $y$, as is typical of shear instability. This enables disturbances on different contours to phase lock with each other. Note the quarter wavelength shift between the upper and lower density interfaces that is characteristic of maximum shear instability (maximises the energy transfer to the perturbation).

As a function of $k a$, the maximum growth rate vanishes as $k a \rightarrow 0$, for very long waves, then rises to a single maximum at $k a \simeq 0.729$ before falling to zero at $k a \simeq 1.094$, beyond which all short wave perturbations are neutrally stable. For comparison, in the case of the uniform buoyancy filament analysed by Juckes (1995), maximum instability occurs at longer wavelengths, $k a_{\max } \simeq 0.52$, and has a growth rate nearly twice as large, $\sigma_{\text {uni }} \simeq 0.203$. Juckes 
(1995) showed that smoothing the edges of the uniform filament has the effect of shifting the instability to shorter wavelengths and slightly reducing its growth rate. The substantial difference in maximum growth rates between the elliptical and uniform filament is likely due to the higher shear inside the uniform filament. By 'elliptical' filament we mean a straight filament with an elliptical buoyancy profile. In the elliptical filament, this shear is uniform and equal to $b_{m} / a$. In the uniform filament, the shear is non-uniform and is found from the derivative of

$$
u_{\text {uni }}=\frac{b_{m}}{a} \ln \left|\frac{y+a}{y-a}\right| .
$$

(see Juckes (1995)). The shear in fact diverges at the edges of the filament. Even at its centre, we find

$$
{\frac{d u_{u n i}}{d y}}_{y=0}=2 \frac{b_{m}}{a}
$$

which is twice as large as in the elliptical filament. This, we believe, explains the nearly twice as large maximum growth rate.

\section{Nonlinear dynamics}

Scott (2011) performed a nonlinear simulation of the elliptical filament perturbed by a single period of a sine wave (not an eigenmode). He confirmed the expected instability and its nonlinear roll up into a billow with a connecting braid, which further rolls up into small billows, etc. Here, we revisit the nonlinear evolution of the elliptical filament, now initiating the perturbation with the most unstable eigenmode and considering two periods in $x$. Like Scott (2011), we use the highly-accurate Contour-Advective Semi-Lagrangian (CASL) algorithm of Dritschel and Ambaum (1997), extended by Dritschel (2011) for the surface QG equations. Here, we use a further refinement, the Combined-Lagrangian Advection Method (CLAM) developed by Dritschel and Fontane (2010). This is the most accurate contour-advection method available. Full details of the standardised numerical parameter settings are provided in Dritschel and Fontane (2010).

The simulation uses a coarse 'inversion' grid of $1024^{2}$ on which the velocity is computed by spectral methods. Buoyancy is represented by material contours, down to scales as small as a 16 th of the inversion grid spacing. The effective resolution is $16384^{2}$. We use a doubly-periodic domain with equal side lengths of $2 \pi$. To accommodate two periods, we then take the filament half width to be $a \simeq 0.364$. The maximum buoyancy $b_{m}$ is set to 1 .

As in many past studies of filament instability, the filament first rolls up into coherent billows connected together by braids (see figure 3). These braids are continuously stretched by the rotating billows. In the case of vorticity in two-dimensional flow, the braids largely thin and become increasingly passive (Dritschel et al (1991)). However, as noted previously by Scott (2011) as well as by Harvey and Ambaum (2010), buoyancy braids are increasingly unstable as they become thinner, despite the large scale strain induced by the billows. This means that the braids subsequently roll up, and the braids between these smaller billows also roll up. But, unlike in the case of a uniform buoyancy filament, here the primary braids have much weaker maximum buoyancy than the billow cores, so the secondary roll up of the braids is less intense. The braids carry relatively low-lying buoyancy, and therefore feel greater relative strain. Nevertheless, even the secondary braids roll up, and it is likely that this continues to successively smaller scales (see Scott (2011)). This results in great complexity at late times, as illustrated in figure 4 at $t=80$ (with two successive zooms). Whether or not scales collapse to zero in finite time is still an open question, though this can occur for a uniform buoyancy braid (see Scott and Dritschel (2014)). 

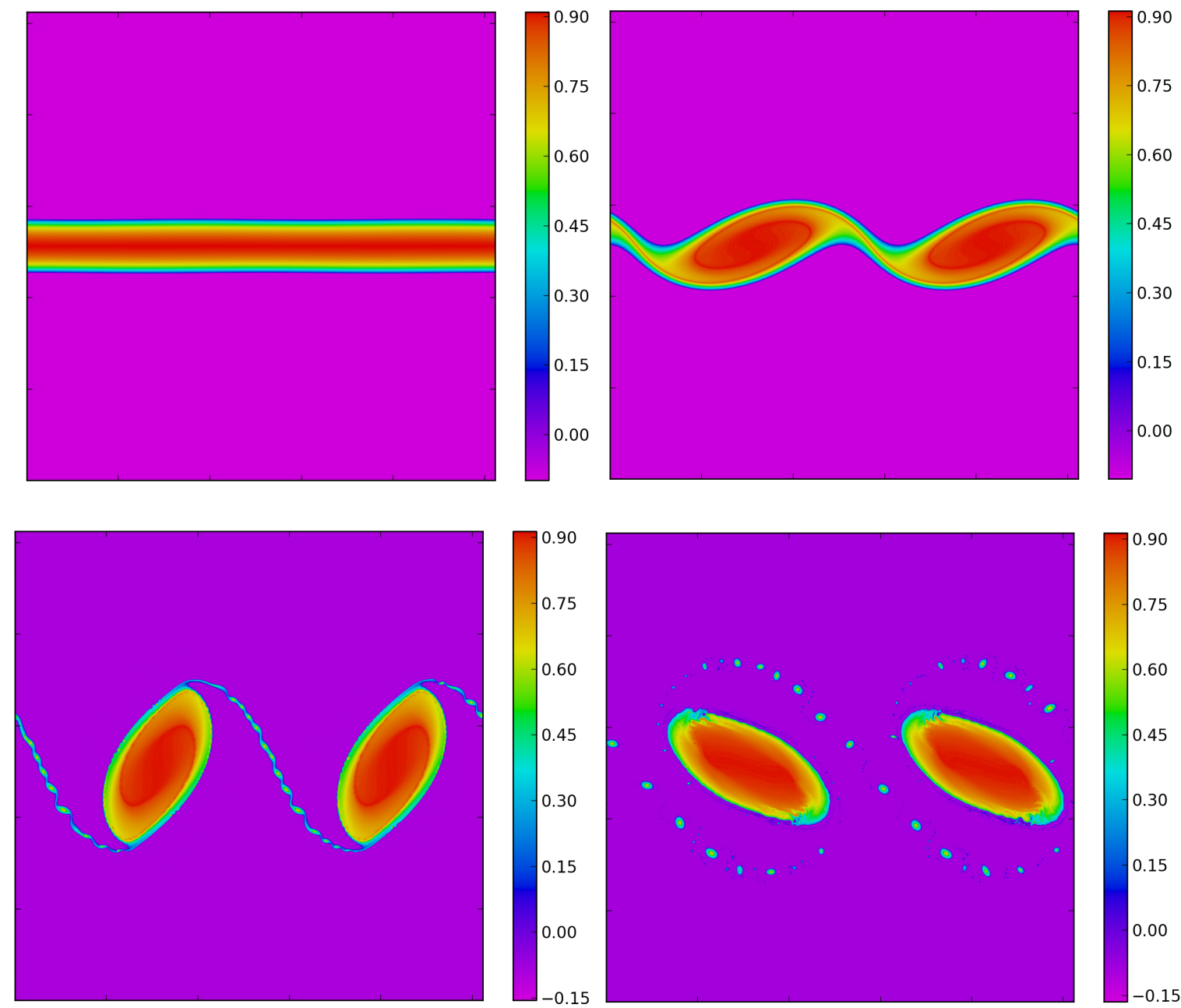

Figure 3. Nonlinear evolution of a buoyancy filament with an elliptical profile. The filament is initially perturbed with the most unstable eigenmode with $k a=0.729$ over two periods in the $x$-direction. Images correspond to $t=0,23,28$, and 30 .
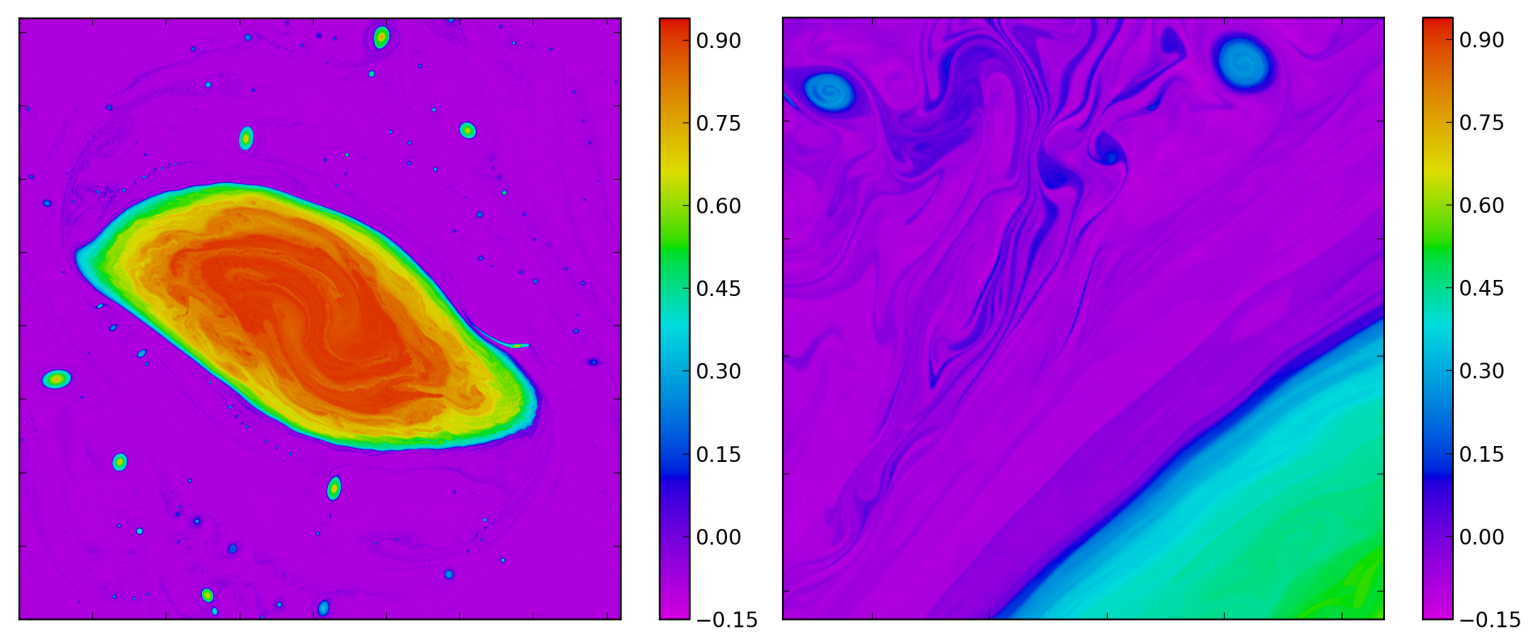

Figure 4. Late-time buoyancy field starting from an elliptical profile, here at $t=80$. Left: zoom into the domain $[0, \pi] \times[-\pi / 2, \pi / 2]$ within the overall $2 \pi \times 2 \pi$ domain. Right: further zoom into the domain $[\pi / 8,3 \pi / 16] \times[21 \pi / 32,23 \pi / 32]$. 
In this section, we have considered only the dynamics at the surface $z=0$. However, there is an influence below which would be felt by any sub-surface structure, such as the internal vortex considered in the following section. Notably, the longest wavelengths on the buoyancy filament penetrate deepest. This is analysed in Appendix 2 in terms of the decay of the buoyancy field $b=\partial \psi_{s} / \partial z$ with depth for the basic-state elliptical filament. We show that the buoyancy contained in the horizontal wavenumber $k_{h}=\sqrt{k^{2}+l^{2}}$ decays as $e^{k_{h} z}(z<0)$, where $k$ and $l$ are the longitudinal and meridional wavenumbers respectively.

\section{Interaction between the filament and an internal vortex}

\subsection{Flow geometry}

We next turn our attention to the interaction between the surface buoyancy filament studied above and an internal vortex. The vortex provides a specific perturbation which in general excites a number of unstable modes on the filament. In return, the filament moves and deforms the vortex. We start with a spherical internal vortex of uniform potential vorticity $q$ for simplicity. We take the radius $r$ of the sphere to be $a$, equal to the half width of the filament so that the two structures have the same length scale (other radii have been considered and their effects are briefly discussed below). The vortex is centred at a depth $2 H$ below the surface, and is horizontally offset from the filament by a distance $2 \rho$. The computational domain is doubly-periodic horizontally with dimensions $2 \pi \times 2 \pi$. In the vertical direction, it is necessary to use a finite depth in the numerical simulations, and here we take the depth to be $2 \pi$ also (in the $N / f$ stretched reference frame). This depth well approximates an infinite depth, to within an error of approximately $e^{-2 \pi}$ (see Appendix 2).

With a second boundary at $z=-2 \pi$, it is necessary to add another boundary condition to be able to solve for the streamfunction $\psi$. As is commonly done, we take the bottom surface to be isothermal (zero buoyancy), implying $\partial \psi / \partial z=0$ there. The alternative is to prescribe no flow $(\psi=0)$ there or to impose a zero vertical velocity, but we have not examined the impact of these options.

A schematic of the flow is presented in figure 5. To keep the effects of periodicity in the horizontal directions as small as practically possible, we chose a small filament half width and vortex radius, both equal to $a=0.25$. Moreover, we have limited $\rho$ to the range $[0,0.6]$ and $H$ to the range $[0.13,1]$.

\subsection{Numerical method}

The numerical method used is described in Perrot et al. (2010). It is an extension of CASL to include the surface buoyancy dynamics, with special care to focus resolution near the upper boundary where the flow is expected to be most complex. The domain is discretised in the vertical direction by 1024 horizontal layers. To find the velocity field from the surface buoyancy and the interior PV contours, they are first converted onto fine horizontal grids with a resolution of $1024^{2}$ using a fast-fill algorithm (Dritschel and Ambaum (1997)). The interior PV field $q$ is then averaged to a coarser grid of $256^{3}$, where it is inverted (using FFTs and spectral differentiation) to find the contribution $\psi_{i}$ to the total streamfunction and hence to the velocity field $u_{i}=-\partial \psi_{i} / \partial y$ and $v_{i}=\partial \psi_{i} / \partial x$. This exploits the fact that the velocity field is typically of broader scale than $q$. However, at and near the upper boundary, we use the finer $1024^{2}$ horizontal grid in the uppermost 4 layers to find the velocity field $u_{s}$ and $v_{s}$ due to the surface buoyancy $b$. This anticipates the finer scales of motion typically associated with the dynamics of surface buoyancy, and significantly improves accuracy. Note: the spectral forms of $u_{s}$ and $v_{s}$ are obtained analytically at each height $z$ from the Fourier coefficients of $b$ (see 
Perrot et al. (2010) and Appendix 2).

To control the inevitable scale cascade of $b$ and $q$, contour surgery is carried out every few time steps at $1 / 16$ th of the inversion grid resolution (as is conventional, see Fontane and Dritschel (2009)). We keep track of the maximum strain on contours, the maximum of $S=\left|\mathbf{u}\left(\mathbf{x}_{j+1}\right)-\mathbf{u}\left(\mathbf{x}_{j}\right)\right| /\left|\mathbf{x}_{j+1}-\mathbf{x}_{j}\right|$ over all nodes $j$, and perform surgery and node redistribution when the time integral of $S_{\max }$, from the last time these operations were done, exceeds 1.5. This is the only form of dissipation occurring in CASL numerical simulations, and is much weaker than in conventional simulations using e.g. hyperviscosity (see e.g. Dritschel and Tobias (2012) and references).

The time integration is carried out using a Runge-Kutta fourth order scheme, with a time step $\Delta t$ adapted to resolve the maximum strain on contours. Specifically, we choose $\Delta t=$ $\min \left(0.2 / S_{\max }, 0.1 \pi / \zeta_{\max }\right)$, where $S_{\max }$ is the maximum straining rate along a contour and $\zeta_{\max }$ is the maximum relative vertical vorticity at the surface. There is no CFL restriction and no numerical instability associated with choosing a larger time step. The time step is chosen purely to maintain high accuracy (see Fontane and Dritschel (2009) for further discussion).

\subsection{Parameter space}

Four dimensionless parameters control the interaction between the surface buoyancy filament and the internal vortex. The first is the ratio of their widths, which we take to be unity except in a couple simulations briefly discussed below. The second is the ratio of their intensities, governed by the parameter $\Lambda=b_{m} /(a q)$. This is the ratio of the shear inside the buoyancy filament to the uniform PV within the vortex. As we shall see below, the interaction depends critically on the sign of $\Lambda$. Without loss of generality, we set the PV within the vortex to be $q=2 \pi$, a positive value. Hence, the sign of $\Lambda$ is dictated by that of $b_{m}$. Positive $b_{m}$ or $\Lambda$ corresponds to clockwise (or negative) rotation of a localised buoyancy anomaly, and vice versa. Hence $b_{m}>0$ means that the buoyancy rotates in the opposite direction to the internal vortex, a situation called 'adverse shear', whereas $b_{m}<0$ corresponds to 'cooperative shear' (Dritschel (1989)). The remaining two dimensionless parameters are $\rho / a$, the ratio of the horizontal offset $2 \rho$ to the filament width $2 a$, and $H / a$, the ratio of the vertical offset $2 H$ to $2 a$.

\subsection{Time evolution}

We first examine the flow evolution in two contrasting situations, with $\Lambda=-4$ and $\Lambda=+4$. In both cases, the vortex starts directly beneath the filament $(\rho=0)$ at a depth $2 H=1$, corresponding to $H / a=2$. The cooperative shear case with $\Lambda=-4$ is shown in the top panels of figure 6 . There are three noticeable characteristics of the flow. First, the velocity field induced by the internal vortex causes the filament to rapidly roll up into billows. This is expected as the filament itself is unstable. Second, one large billow forms on the top of the internal vortex. Finally, the internal vortex is only moderately deformed by the shear induced by the surface buoyancy filament. By contrast, the evolution is very different for the adverse shear case with $\Lambda=+4$, shown in the bottom panels of figure 6 . The rapid billow formation is the only similar characteristic, but here no billow forms over the internal vortex. Moreover, the shear induced by the filament on the vortex pulls the vortex into a thin filament. This is consistent with the greater destructive capability of adverse shear in vortex interactions (Kida (1981), Dritschel (1990), Legras and Dritschel (1993), Trieling, Dam and van Heijst (2010)).

The adverse shear not only stretches the vortex into a filament, but prevents a billow from forming over the vortex initially. The shear induced by the vortex is largest above it and, as it opposes the shear of the filament there, it results in a stagnation point at the centre of 


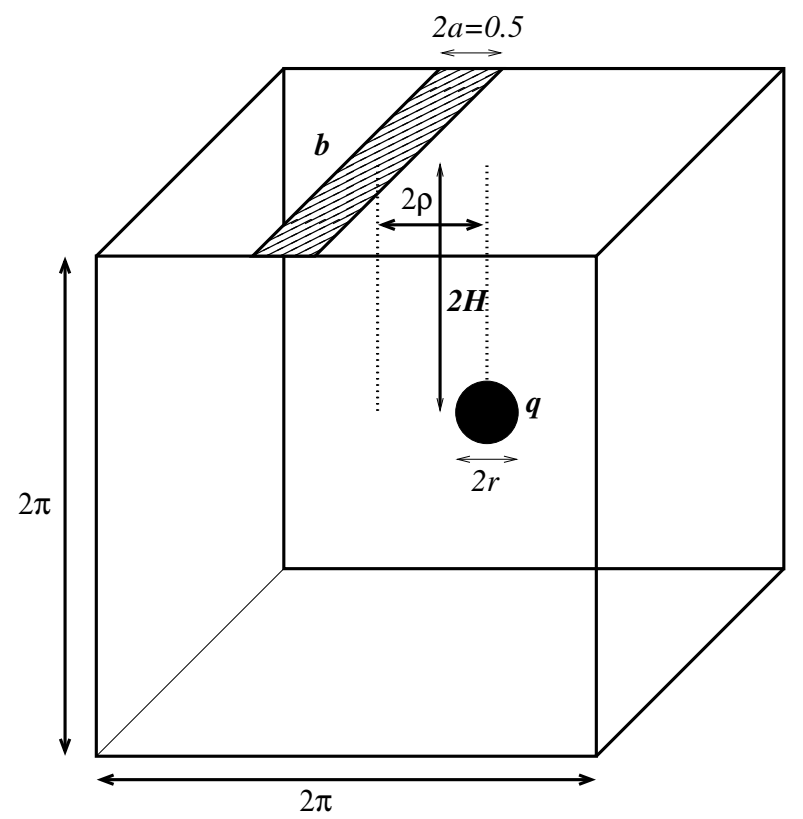

Figure 5. Flow geometry illustrating the initial conditions for a surface buoyancy filament and an internal vortex.
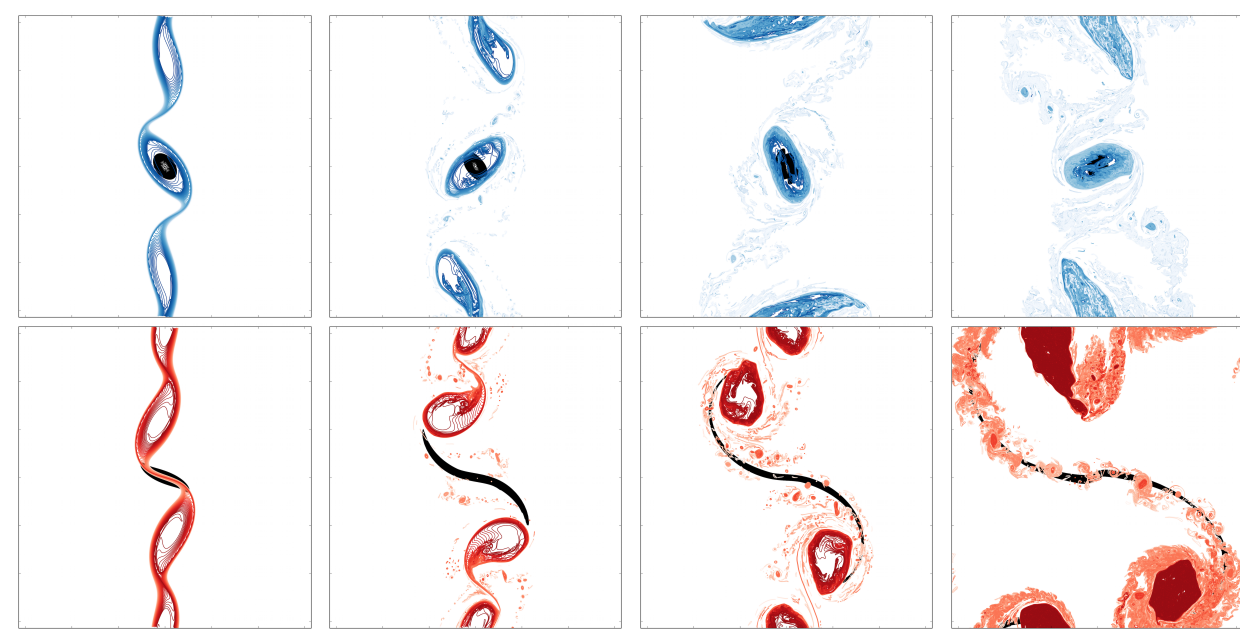

Figure 6. Surface buoyancy and PV evolution for $\rho / a=0, H / a=2$ and $\Lambda=-4$ (top) and $\Lambda=4$ (bottom). Buoyancy contours are rendered in blue for $b<0$ and in red for $b>0$. PV contours (in the interior) are rendered in black. The view is from the top, looking down into the flow. The times displayed are $t=2.5,5,7.5$, and 10 (top), while they are $t=2.5,5,7.5$, and 12.5 (bottom). The surface buoyancy filament is represented by 50 levels of buoyancy iso-value (contours) equally spaced in $\left(0, b_{m}\right)$.

the filament directly above the vortex. This flow structure causes the filament to thin fastest above the vortex, inducing billows on either side of it. By contrast, cooperative shear results in a centre (an elliptical flow region) at the surface above the vortex. This induces a large billow to form directly above the vortex, as observed.

For both cases illustrated in figure 6, the surface buoyancy field becomes highly intricate. Braids between billows roll up. Other billows merge and split, and the braids between those billows roll up into smaller billows, and so on, resulting in a rapid growth in flow complexity. There are scale cascades occurring in both directions, scale growth through billow formation and merging on the one hand, and scale reduction through the creation and stretching of braids at ever smaller scales. 


\subsection{Parameter dependence}

We next investigate more generally how the flow evolution depends on the parameters of the initial flow state, $\Lambda, \rho / a$ and $H / a$.

We begin by studying the influence of $H / a$ keeping $\rho / a=0$ and $\Lambda= \pm 2$ - again both cooperative and adverse shear are considered. Varying $H / a$ has two main effects. On the one hand, it controls the amplitude of the flow induced by the vortex on the filament. Notably however the growth of disturbances on the filament after the initial perturbation by the vortex occurs at a rate proportional to the shear within the filament, $b_{m} / a$. This can be much greater than the shear induced by the vortex, which may be estimated to be $\sim q(H / a)^{-3}$, noting that the velocity field external to a spherical vortex decays like $(a / r)^{2}$ where $r$ is the distance from the vortex centre. On the other hand, decreasing $H / a$ increases the shear induced by the filament on the vortex. The initial maximum horizontal shear induced by the filament can be calculated semi-analytically from the surface buoyancy distribution at $t=0$. Then $b=b(y, z)$ and

$$
\frac{\partial u}{\partial y}=-\frac{\partial^{2} \psi_{s}}{\partial y^{2}}=\frac{\partial^{2} \psi_{s}}{\partial z^{2}}=\frac{\partial b}{\partial z}
$$

using the fact that $\psi_{s}$ is a harmonic function. After Fourier transforming the surface buoyancy $b(y, 0)$ in $y$, we can obtain the interior shear directly from

$$
\frac{\partial \hat{u}_{l}}{\partial y}=\frac{\partial \hat{b}_{l}}{\partial z}=\frac{e^{l z}+e^{-l(4 \pi+z)}}{1-e^{-4 \pi l}} l \hat{b}_{l}(z=0)
$$

where $\hat{b}_{l}(z)$ is the Fourier coefficient of $b(y, z)$ and $l$ is the $y$ wavenumber (see Appendix 2). Notably, only the coefficients of $b$ at the surface are required.

Contours of the dimensionless shear directly below the filament, $\gamma=q^{-1} \partial u(y=0, z) / \partial y$, are plotted in figure 7 as a function of $\Lambda$ and $H / a$. A priori, one would expect this shear, if adverse, to overwhelm the vortex if it exceeds $0.0768 q$ (based on the behaviour of an initially spherical QG ellipsoid subject to steady uniform horizontal shear, see McKiver and Dritschel (2003)). This criterion is seen to work well for vortices subject to adverse shear. In general, cooperative shear is non-destructive, though it can lead to tearing of filaments from the vortex, as seen here when $|\gamma|$ is large.

The dependence of the flow evolution on the depth of the vortex $H / a$ is shown next in figure 8, again for both cooperative and adverse shear, all at a fixed identical time, $t=10$. The approximate criterion $\gamma>0.0768$ divides the two cases on the left where the vortex is ripped apart from the two cases on the right where it remains intact. For strong cooperative shear at small $H / a$ (top left panel), the periphery of the vortex is torn away leaving a reduced vortex core below the surface buoyancy billow that forms in the centre of the domain. As expected, in all cases for both the cooperative and adverse shear, the buoyancy filament breaks into billows. At the time shown, the billow formation is more advanced for decreased $H$. Recall that in the linear stage the amplitude of disturbances on the unstable filament grows as $A e^{\sigma t}$, where $A$ is the initial amplitude of the perturbation and $\sigma$ the growth rate. The effect of decreasing $H / a$ is roughly equivalent to increasing $A$; hence, a given disturbance amplitude will be reached earlier for decreasing $H / a$. We also see that when the vortex is near the surface, it breaks into thin filaments (primarily in the adverse shear cases). These PV filaments become weaker dynamically as they thin, by contrast to the surface buoyancy filaments. As a result, the evolution of the PV filaments is increasingly dictated by the flow generated by the breaking buoyancy filament. In the most extreme case shown $(H / a=0.52)$, even in the adverse shear case the PV filaments appear to follow the swirling patterns of the billows generated from the buoyancy filament. By contrast, for weaker shear (larger $H / a$ ) the internal PV is more able to remain compact and dynamically active, and tends to reside away 


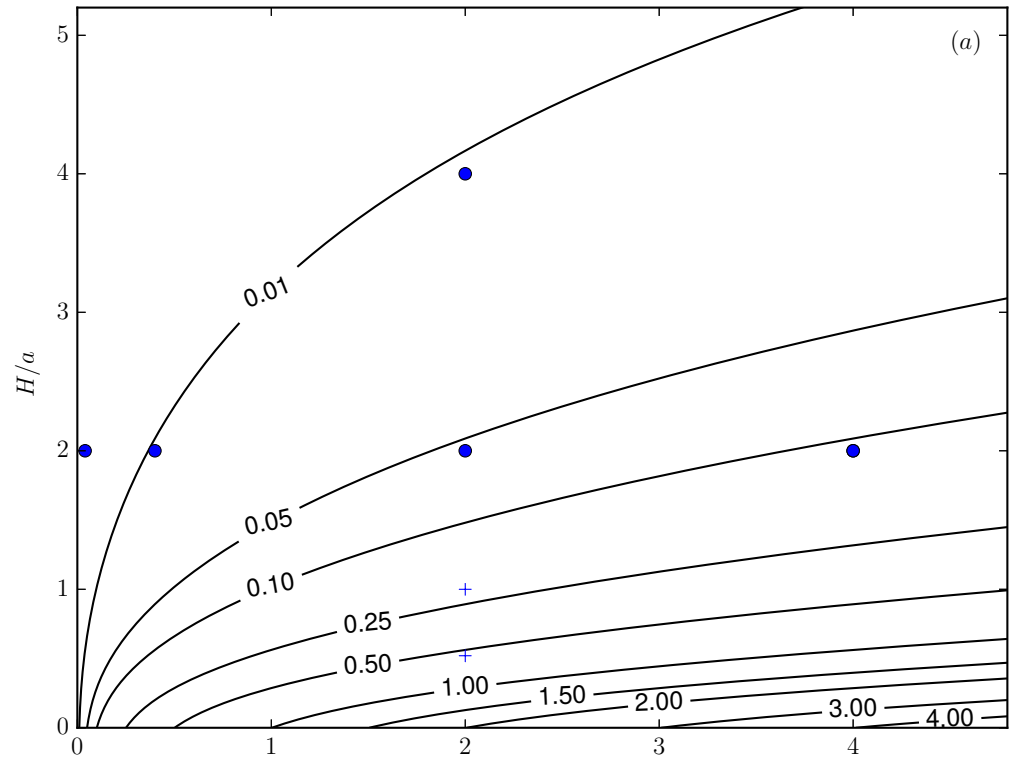

$|\Lambda|$

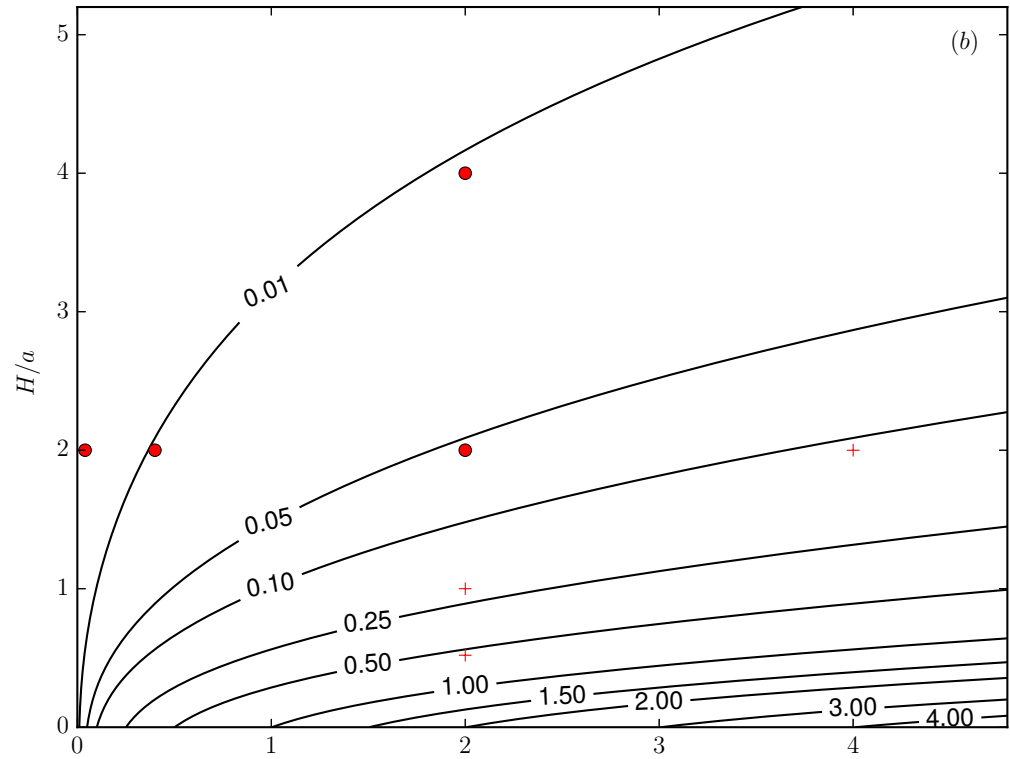

$|\Lambda|$

Figure 7. Magnitude of the shear induced by the filament scaled by the PV of the vortex, $|\gamma|$, as a function of $|\Lambda|$ and $H / a$. Markers are placed in the diagram corresponding to the numerical experiments for $(a)$ cooperative shear with $\Lambda<0$, and $(b)$ adverse shear with $\Lambda>0$. A circle $(\bullet)$ indicates that the vortex remains ellipsoidal. A plus sign $(+)$ indicates that the vortex has been either partially or completely strained out. This confirms that $\gamma>0.0768$ provides a fair estimate of the shear required for a strong deformation of the internal vortex subject to adverse shear.

from the buoyancy billows.

We next study the influence of $\Lambda$, keeping $\rho / a=0$ and $H / a=2$. Since the characteristics of the vortex $(r$ and $q)$ are fixed, by also fixing the location of its centre $(\rho, H)$, the flow initially induced by the vortex on the filament is the same in all cases. Varying $\Lambda$ however changes the rate at which the filament destabilises and rolls up into billows. Taking this into account, figure 9 shows the flow state at the equivalent 'buoyancy time', $\tau=t a /\left|b_{m}\right|=5 / 8=0.6125$. For the reference case, this corresponds to $t=2.5$ while for the smallest $|\Lambda|$, it corresponds to $t=250$, a time by which the deep vortex has rotated over 80 times. As a consequence, the weak buoyancy filament forms a tight spiral which rolls up into many small billows. 

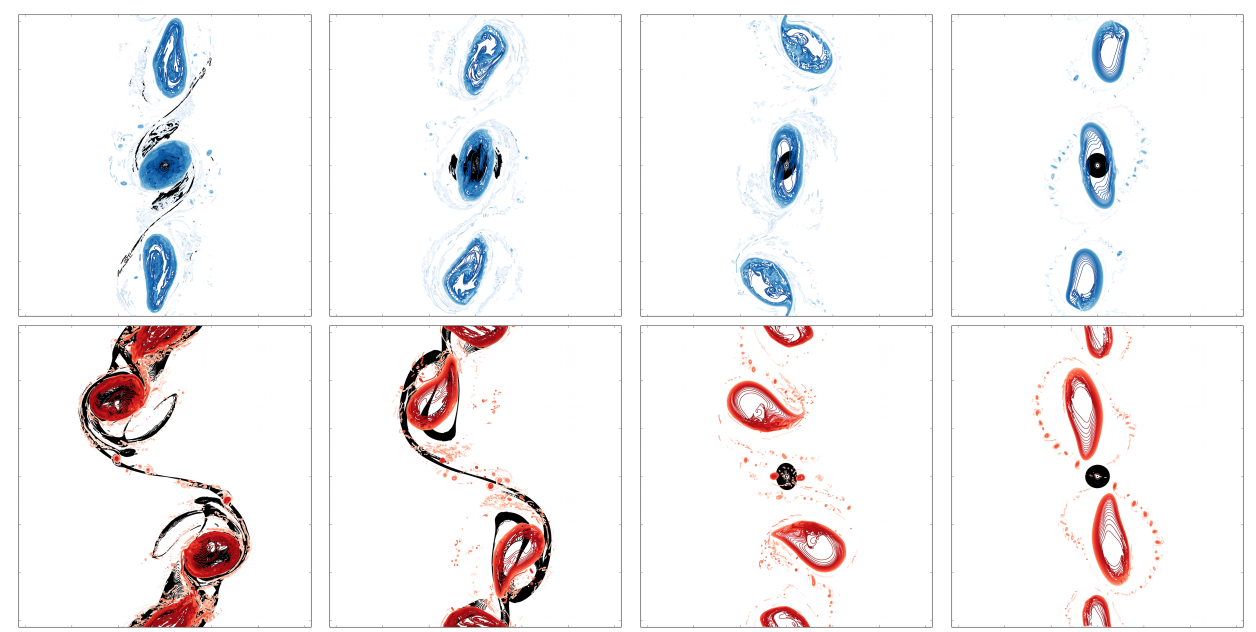

Figure 8. Surface buoyancy and PV contours at $t=10$ for $\rho / a=0, \Lambda=-2$ (top) and $\Lambda=2$ (bottom) for various values of the dimensionless vertical offset $H / a$. From left to right $2 H / a=0.52,1,2$, and 4 . The surface buoyancy filament is represented by 50 levels of buoyancy iso-value (contours) equally spaced in $\left(0, b_{m}\right)$.
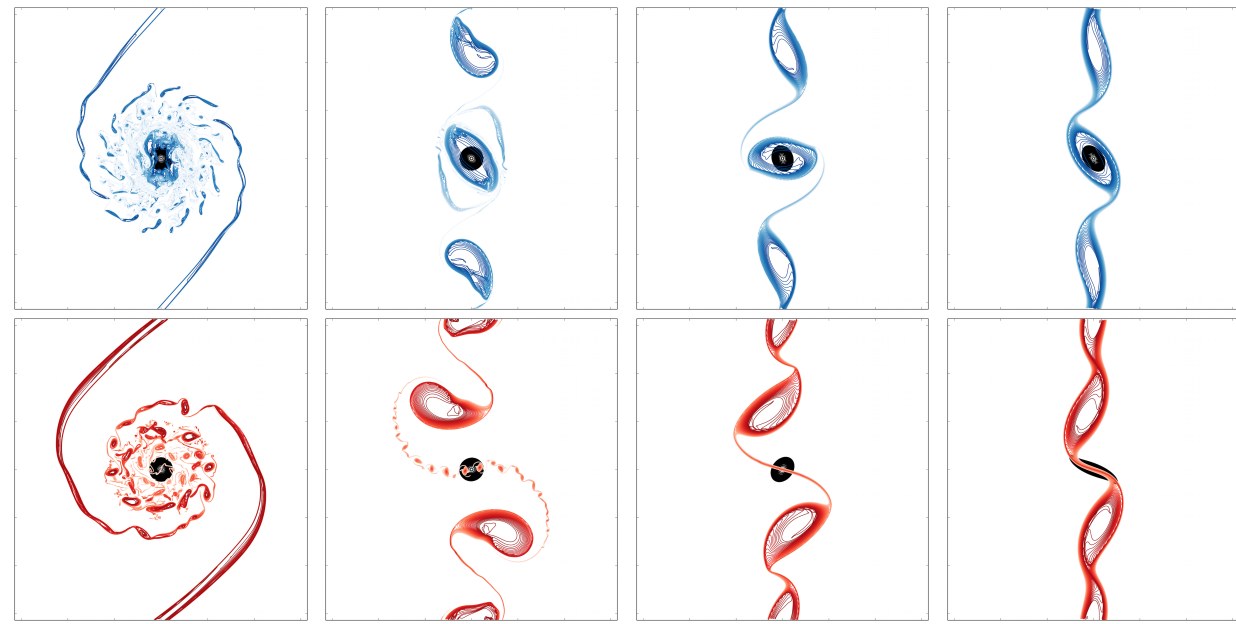

Figure 9. Surface buoyancy and PV contours for $\rho / a=0, H / a=2$ and from left to right $\Lambda= \pm 0.04$ at $t=250$, $\Lambda= \pm 0.4$ at $t=25, \Lambda= \pm 2$ at $t=5$ and $\Lambda= \pm 4$ at $t=2.5$. Top: cooperative shear with $\Lambda<0$; bottom: adverse shear with $\Lambda>0$. The surface buoyancy filament is represented by 50 levels of buoyancy iso-value (contours) equally spaced in $\left(0, b_{m}\right)$.

Initially, the filament instability is suppressed by the straining induced by the deep vortex. This straining comes from the decrease in rotation speed of the vortex flow with horizontal distance from the vortex centre. But, as the filament scale reduces, the potential for instability increases. Eventually, the shear within the filament, which scales with $b_{m}$ divided by the local filament half width $a(t)$, is great enough to overcome the strain induced by the vortex below it and billows form. Harvey and Ambaum (2010) have found for example that the minimum strain needed to stabilise the uniform-buoyancy filament is

$$
s>0.0371 \frac{b_{m}}{a},
$$

This threshold keeps increasing as $a(t)$ decreases, so eventually the limited vortex strain will be insufficient to stabilise the buoyancy filament. That is, the weaker the filament (the smaller $\Lambda$ is), the smaller $a(t)$ must be before instability and roll up occurs. Essentially, the local shear inside the filament, $b_{m} / a(t)$, must exceed the roughly fixed straining induced by the vortex. The exception is the billow formation above the vortex in the cooperative shear case. 


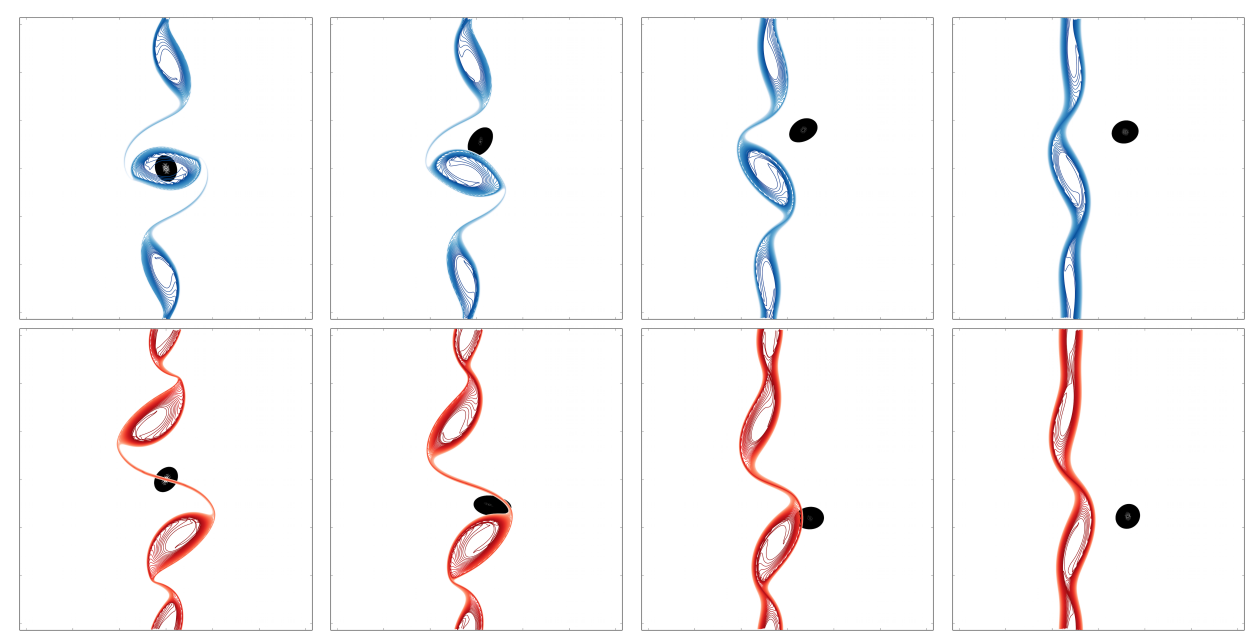

Figure 10. Surface buoyancy and PV contours at $t=5$ for $\Lambda= \pm 2, H / a=2$ and from left to right $\rho / a=0,0.8,1.6$, and 2.4. Top: cooperative shear with $\Lambda<0$; bottom: adverse shear with $\Lambda>0$. The surface buoyancy filament is represented by 50 levels of buoyancy iso-value (contours) equally spaced in $\left(0, b_{m}\right)$.
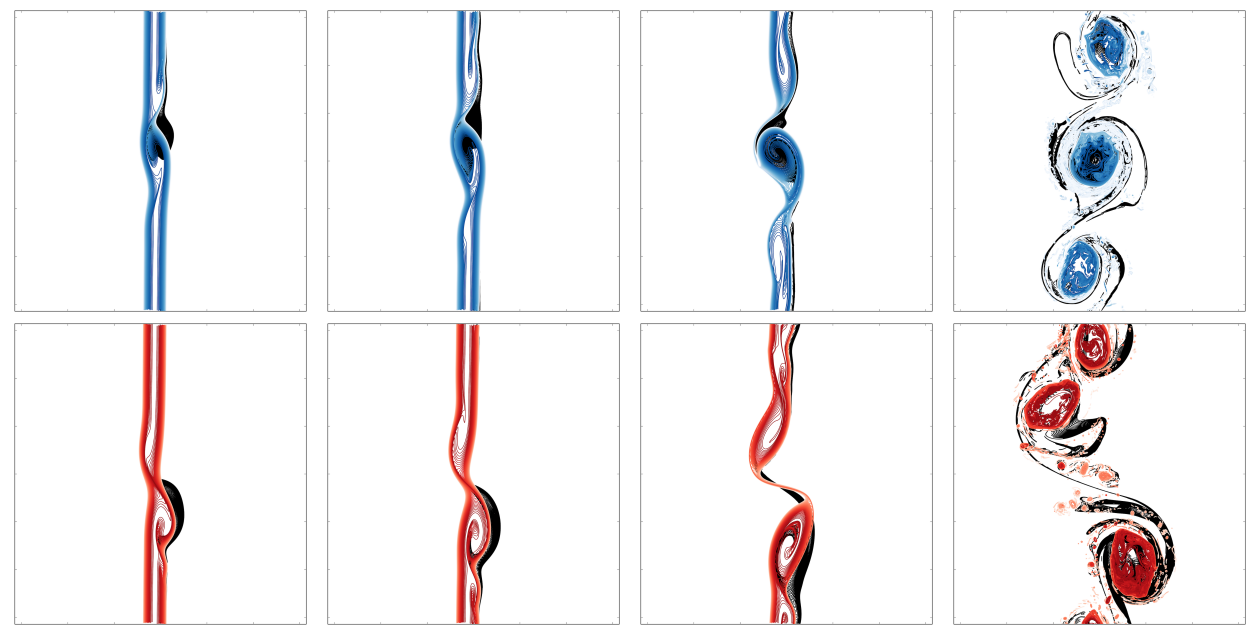

Figure 11. Surface buoyancy and PV contours for $\Lambda= \pm 2, \rho / a=0.5, H / a=0.52$ and from left to right at $t=1,1.5$, 2.5 and 10. Top: cooperative shear with $\Lambda<0$; bottom: adverse shear with $\Lambda>0$. The surface buoyancy filament is represented by 50 levels of buoyancy iso-value (contours) equally spaced in $\left(0, b_{m}\right)$.

Otherwise, around the vortex the filament is twisted into a spiral and thinned until its shear becomes sufficiently intense to form further small-scale billows. Even the larger $\Lambda$ cases for adverse shear show some suppression of filament roll up above the vortex, but as the vortex induced straining weakens with horizontal distance, there is less stretching of the filament and a correspondingly more rapid growth of the filament instability, resulting in larger billows.

We next study the influence of the dimensionless horizontal offset $\rho / a$, keeping $\Lambda= \pm 2$ and $H / a=2$. Four increasing values of the offset are considered (from left to right) in figure 10. There are three main effects. First, for $\rho / a>0$ the vortex centre is no longer centred at a point of zero velocity, so it is advected by the velocity field induced by the filament. Second, the straining flow induced by the vortex on the filament is no longer symmetric. Moreover, this asymmetry is enhanced by the fact that the vortex propagates and therefore changes where it most strongly disturbs the filament. Third, as the initial horizontal offset between the vortex and the filament increases, the interaction between the two weakens. This is expected since the straining due to the vortex diminishes with the cube of the distance between the vortex and the centre of the filament. 

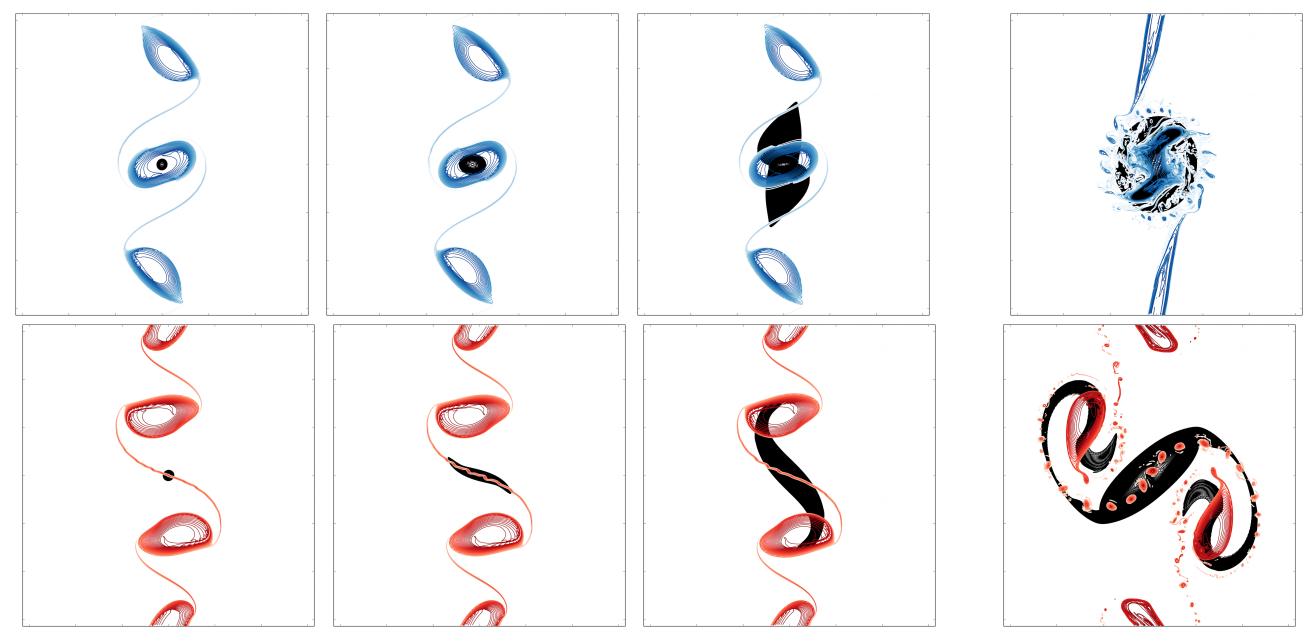

Figure 12. Effect of vortex size. Top: cooperative shear with $\Lambda<0$; bottom: adverse shear with $\Lambda>0$. In all cases $H / a=2$ with $a=0.25$. Results are shown at the dimensionless time $t_{q}=2 \pi t / q=3$. From left to right, first column: $\Gamma_{v}=2 \pi^{2} / 24, \Lambda= \pm 0.4$; second column: $\Gamma_{v}=\pi / 48, \Lambda= \pm 4$; third column: $\Gamma_{v}=2 \pi^{2} / 24, \Lambda= \pm 40$; last column: $\Gamma_{v}=512 \pi^{2} / 375, \Lambda= \pm 4$. The surface buoyancy filament is represented by 50 levels of buoyancy iso-value (contours) equally spaced in $\left(0, b_{m}\right)$.

An interesting sub-case occurs when the vortex is located just below the surface with its centre lying directly below the edge of the filament $(\rho / a=0.5)$. This propels the vortex at the fastest possible rate initially. Two examples (for cooperative and adverse shear) are illustrated in figure 11. In these examples, the vortex is strongly sheared and pushed along by the velocity field induced by the filament. The vortex then rapidly disintegrates into a myriad of small-scale vortices and filaments which are swept around the billows formed by the unstable filament.

Finally, we investigate the effect of varying the vortex radius $r$ relative to the filament half width $a$. We keep $a=0.25$ as well as $H / a=2$, but now vary $r$ and/or $q$. In the first set of numerical experiments, we fix the strength of the vortex $\Gamma_{v}=\iiint_{\text {vortex }} q d V=$ $4 \pi q r^{3} / 3= \pm \pi^{2} / 24$, the same as in the reference case for which $r=0.25$ and $q=2 \pi$. Keeping $b_{m}= \pm 2 \pi$, we consider $\Lambda=b_{m} /(a q)= \pm 0.4, \pm 4$ and \pm 40 , adapting $r$ to keep $\Gamma_{v}= \pm \pi / 48$. This gives $r \approx 0.116,0.25$ and 0.539 , respectively. The flow structure at the dimensionless time $t_{q}=2 \pi t / q=3$ is presented in figure 12 (left 3 columns). We see that, at least over this range of $r$, the vortex size has virtually no influence on the evolution of the buoyancy filament. The strip destabilises according to the most amplified mode consistent with the imposed periodicity. By contrast, if we increase the vortex radius to $r=0.8$ while keeping $q=2 \pi$ (and therefore $\Lambda= \pm 4$ ), the vortex is substantially stronger $\left(\Gamma_{v}=512 \pi^{2} / 375\right.$, which corresponds to $r / a=3.2$ ) and thereby exerts greater strain on the buoyancy filament. This is illustrated in the right column in figure 12. For cooperative shear (top), the vortex wraps the filament into a spiral which is just beginning to destabilise. For adverse shear, the core of the vortex consolidates after shedding substantial filaments, which roll up underneath the buoyancy billows. Above the vortex (and parts of the ejected filaments), the braid connecting the innermost buoyancy billows itself rolls up into many smaller billows.

\subsection{Energetics}

We next examine the distribution of energy within the flow. The surface buoyancy variance spectrum

$$
\mathcal{B}\left(k_{h}\right)=\sum_{\sqrt{k^{2}+l^{2}}=k_{h}}\left|\hat{b}_{k, l}\right|^{2}
$$



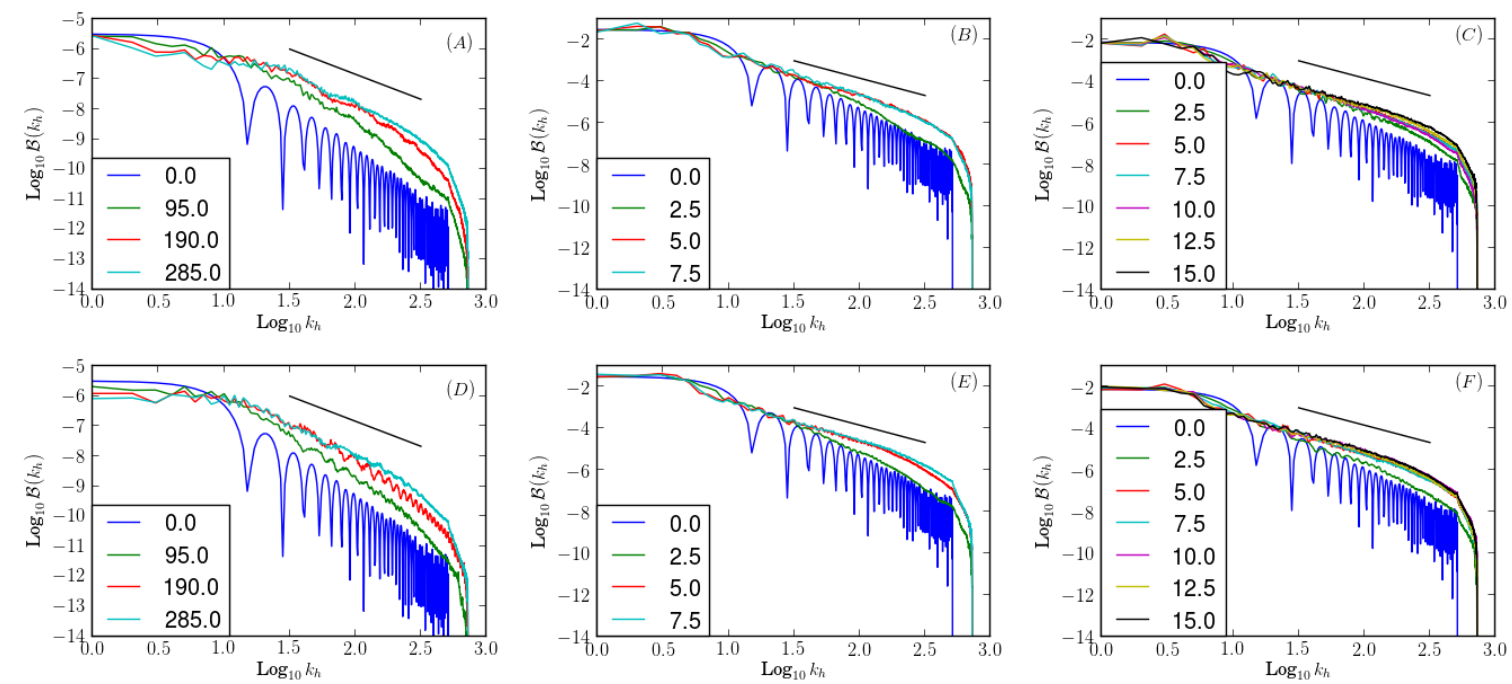

Figure 13. Surface buoyancy spectra $\mathcal{B}\left(k_{h}\right)$ for $\rho / a=0$ at the times indicated. $(A): \Lambda=-0.04, H / a=2$. $(B): \Lambda=$ $-4, H / a=2 .(C): \Lambda=-2, H / a=0.52$. $(D): \Lambda=0.04, H / a=2 .(E): \Lambda=4, H / a=2 .(F): \Lambda=2, H / a=0.52 . \mathrm{A}$ reference line for $k_{h}^{-5 / 3}$ is indicated.

is shown in figure 13 for several choices of flow parameters. Here, we see how the nonlinear dynamics redistributes buoyancy with scale $k_{h}$ (the horizontal wavenumber). Recall that in surface quasi-geostrophy, this variance equals the surface 'self'-kinetic energy

$$
|\hat{b}|^{2}(k, l)=\left|\hat{\mathbf{u}}_{s}\right|^{2}(k, l)
$$

where $\mathbf{u}_{s}$ is the velocity at the surface induced by the buoyancy alone, and a hat denotes a horizontal Fourier transform. It is important to notice that this quantity does not include the part of the surface velocity induced by the interior PV distribution. Hence, it is not the actual kinetic energy at the surface (unless $q=0$ ). However, the surface buoyancy variance spectrum allows one to analyse the energy transfer associated with surface buoyancy alone.

All cases shown in figure 13 exhibit a small to moderate decrease of the buoyancy variance at the largest scales (small $k_{h}$ ). This decrease is accompanied by an increase in the energy at small to intermediate scales. In particular, in the 'inertial range' far from both the domain scale and the grid scale, the spectrum shallows to $k^{-5 / 3}$ in the most turbulent cases examined. This spectral form is consistent with the rigorous mathematical analysis of surface QG energy spectra in Tran, Blackbourn and Scott (2011). The initial $k_{h}^{-3}$ spectrum is due to the singularity in $\partial b / \partial y$ at the edge of the filament. The $k_{h}^{-3}$ spectrum occurring at very high $k_{h}$ is a numerical artefact. At high $k_{h}$, the buoyancy field $b$ is being advected by a flow field which is truncated at the wavenumber $k_{h}=512$ corresponding to the grid resolution. As a result, finer scales in $b$ are partially suppressed for $k_{h}>512$.

We next consider the total, domain-integrated energy of the flow. This is given by

$$
E_{t}=\iiint_{D}\left(u^{2}+v^{2}+b^{2}\right) d x d y d z=\iiint_{D}|\nabla \psi|^{2} d x d y d z,
$$

and is conserved in the absence of diabatic and viscous effects (see Held et al. (1995) and Capet et al. (2008)). We decompose the total energy $E_{t}$ into three parts as follows:

$$
E_{t}=\iiint_{D}\left|\nabla\left(\psi_{i}+\psi_{s}\right)\right|^{2} d V=E_{q}+E_{b}+E_{i}
$$

where

$$
E_{q}=\iiint_{D}\left|\nabla \psi_{i}\right|^{2} d V
$$



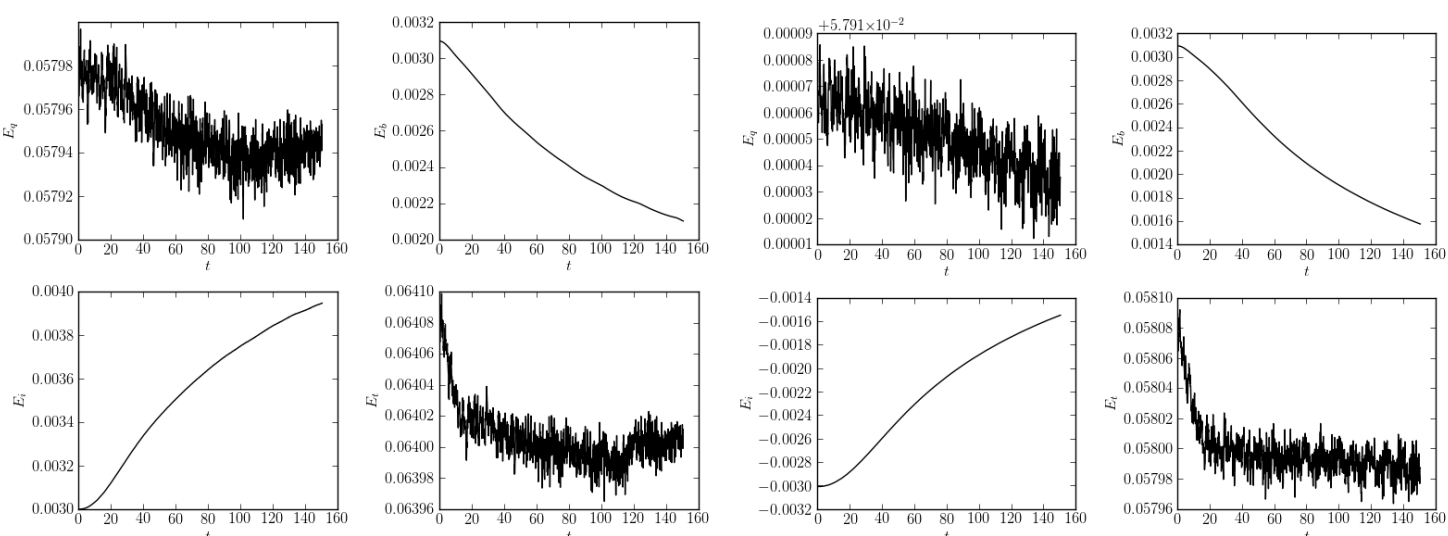

Figure 14. Energy components computed from data on a $256^{3}$ grid for $\rho / a=0, H / a=2$, and $\Lambda= \pm 0.04$. Left, cooperative shear with $\Lambda<0$, and right, adverse shear with $\Lambda>0$. $E_{q}$ is the self energy of the internal vortex, $E_{b}$ is the self energy of the buoyancy filament, $E_{i}$ is the interaction energy, and $E_{t}$ is the total energy.

is the self energy associated with the vortex alone (positive definite),

$$
E_{b}=\iiint_{D}\left|\nabla \psi_{s}\right|^{2} d V
$$

is the self energy associated with the filament alone (also positive definite), and

$$
E_{i}=E_{t}-E_{q}-E_{b}=2 \iiint_{D} \nabla \psi_{i} \cdot \nabla \psi_{s} d V
$$

is the interaction term, which can be either positive of negative depending on whether the interaction is a sink or a source of energy. This term may be interpreted as the correlation between the flow contributions of the buoyancy filament and the vortex. As shown below, it is mainly positive for cooperative shear and negative for adverse shear. This makes sense since the interaction energy is the product of the two velocities $\mathbf{u}_{i}$ and $\mathbf{u}_{s}$, which largely oppose each oin $\left(0, b_{m}\right)$ ther for adverse shear.

Numerically, the horizontal gradients appearing in the expressions above are computed spectrally, while fourth-order compact differencing is used in the vertical direction. The integration is performed by the trapezoidal rule in physical space. Due to the large memory space required for computing $\nabla \psi_{i}$ and $\nabla \psi_{s}$, the integration is performed on the same $256^{3}$ grid used to represent the interior velocity field in the nonlinear simulations. While the surface and near-surface layers are resolved on a grid four times finer, for the purpose of computing the energy components we have truncated the spectra of $\nabla \psi_{i}$ and $\nabla \psi_{s}$ there to 128 total wavenumbers, as in the interior. This means that the energy between wavenumbers 128 and 512 is not accounted for, even though these wavenumbers are used in the nonlinear simulations. This affects only the part of the domain near the upper surface, and then only weakly since the energy contained in these scales is a small portion of the total. Moreover, the contribution to the energy in this range of wavenumbers is only significant within a vertical grid interval $\Delta z=2 \pi / 256$ of the surface.

Results for $\rho / a=0, H / a=2$ and $\Lambda= \pm 0.04$ are presented in figure 14. In these two weak buoyancy cases, the self energy of the internal vortex is almost conserved. This is consistent with the fact that the vortex remains almost undeformed. The overall variation is $\Delta E_{q} \sim$ $10^{-4}$, comparable to the variation in total energy $E_{t}$. On the other hand, the interaction energy exhibits a variation around 10 times larger, and its upward trend is nearly perfectly compensated by the downward trend in the self energy due to the buoyancy filament. Hence, as the filament destabilises, it loses energy to the interaction energy. There is little difference between the adverse and cooperative shear cases in terms of energetics. 

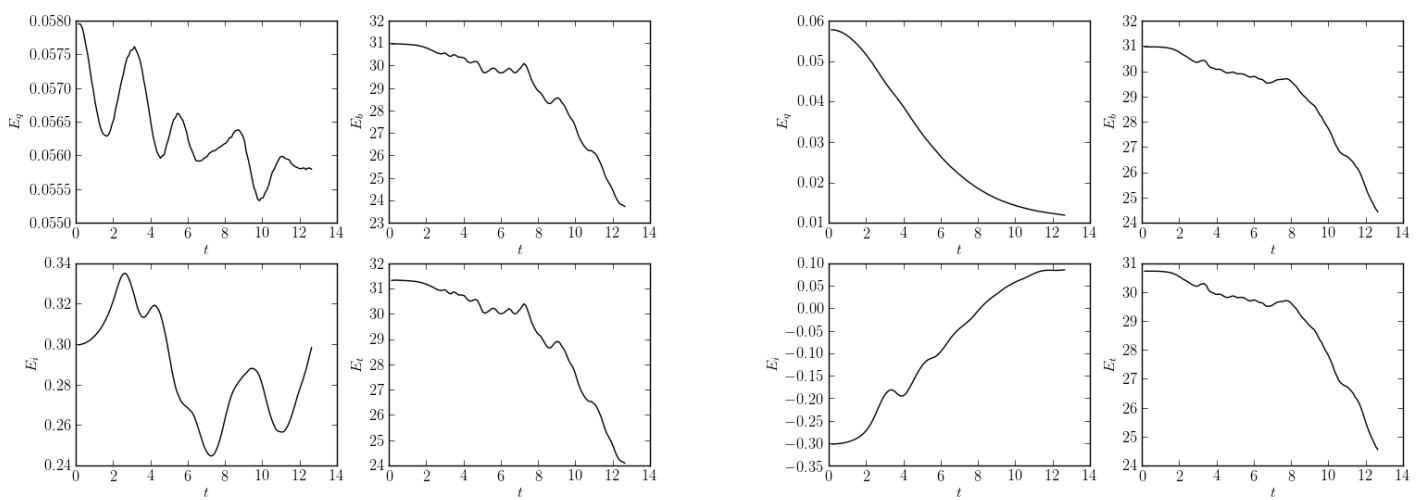

Figure 15. Energy components as in figure 14 but for $\rho / a=0, H / a=2$, and $\Lambda= \pm 2$. Left, cooperative shear with $\Lambda<0$, and right, adverse shear with $\Lambda>0$.

For the same dimensionless horizontal and vertical offsets, $\rho / a=0$ and $H / a=2$, the vortex can be stretched out by increasing the relative intensity of the filament, $\Lambda$. In figure 15 , for $\Lambda= \pm 2$, we show how the various energy components evolve. The corresponding PV and buoyancy evolution is shown in figure 6 . In the adverse shear case, the vortex is overwhelmed by the shear induced by the buoyancy filament. Regarding the energetics, the vortex energy plunges in the adverse shear case, and this is followed by a decay in both the self energy of the buoyancy filament and the total energy (which is dominated by the self energy of the filament). The interaction energy rises significantly, more than the vortex self energy decays. By contrast, in the cooperative shear case, the vortex self energy oscillates and weakly decays. The interaction energy first rises then falls and oscillates - there is no clear trend. Again, there is a decay in both the self energy of the buoyancy filament and the total energy, and again the decrease in self energy of the filament is the dominant process energetically.

The lack of conservation of energy is expected since buoyancy typically exhibits a forward spectral cascade at the surface (Tran, Blackbourn and Scott (2011)), and therefore this cascade inevitably carries energy, in the inviscid limit, beyond the wavenumber truncation used. This is not unlike the behaviour exhibited by three-dimensional homogeneous turbulence, which similarly exhibits a forward energy cascade.

Regarding the vortex self energy $E_{q}$, it exhibits its maximum possible value at $t=0$ when the vortex is a perfect sphere. Any deformation reduces the vortex self energy. The oscillations in $E_{q}$ seen in figure 15 for the cooperative shear case indicate that the vortex first deforms then rebounds close to a spherical form before being further deformed, and so on. This is typical of the way in which cooperative shear acts on vortices (Dritschel (1990)).

One final example illustrates the energy transfers occurring during particularly strong interactions. We consider a vortex near the surface with $H / a=0.52$ with no horizontal offset $\rho / a=0$ and with filament-vortex intensity ratios $\Lambda= \pm 2$. The evolution of the energy components is presented in figure 16. In both cases the vortex is strongly deformed, but recovers somewhat in the cooperative shear case and loses only $10-15 \%$ of its self energy $E_{q}$. No recovery occurs in the adverse shear case - the vortex disintegrates into a myriad of filaments (see lower left panel of figure 8). Here also the interaction energy decreases by $30-40 \%$ in magnitude. The plateau reached from $t=6$ to 18 is likely associated with the passive-tracer-like behaviour of the interior PV as it is swept around by the more energetic surface buoyancy. The situation for cooperative shear is less clear: there are thin filaments of PV ripped from the vortex at early time which subsequently get swept up by the buoyancy field, but a significant part of the PV field remains coherent and mainly concentrated in the original vortex (see upper right panel of figure 8). The self energy of the buoyancy filament $E_{b}$ again dominates 

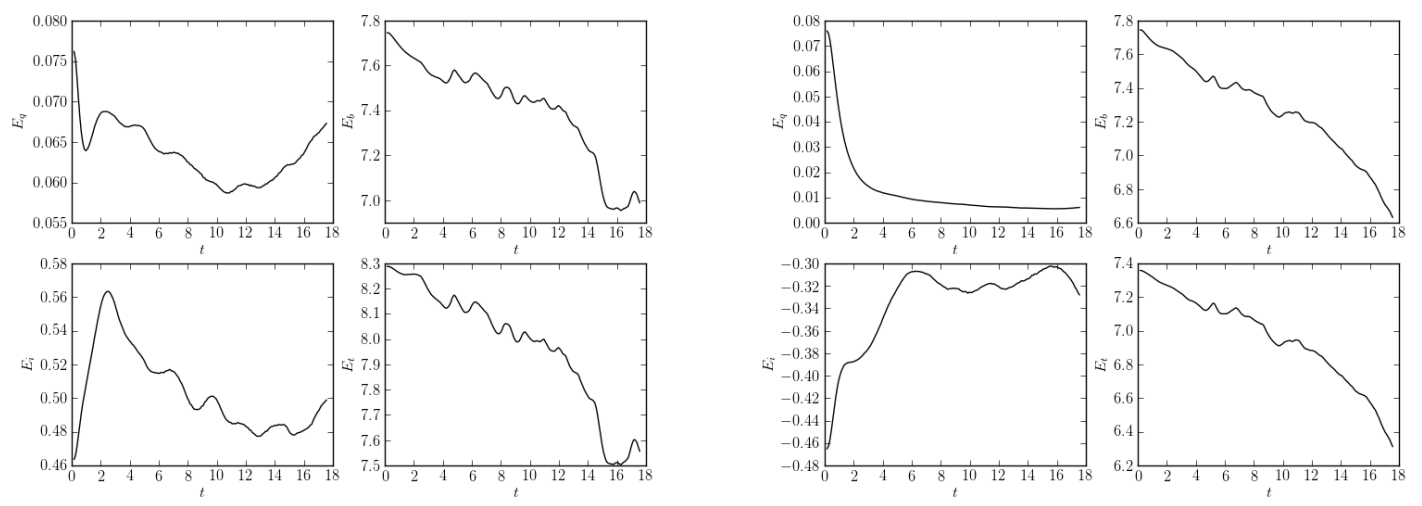

Figure 16. Energy components as in figure 14 but for $\rho / a=0, H / a=0.52$, and $\Lambda= \pm 2$. Left, cooperative shear with $\Lambda<0$, and right, adverse shear with $\Lambda>0$.

the energetics and is largely responsible for the decay in total energy $E_{t}$. The arrest of this decay at late times in the cooperative shear case occurs because the main billows formed early on do not subsequently strongly interact. By contrast, the billows merge in the adverse shear case, leading to a stronger and sustained decay in $E_{b}$ and $E_{t}$.

\section{Conclusion}

This paper has studied the stability of a surface buoyancy filament having uniform shear, and has examined its complex nonlinear interaction with an internal vortex. We have explicitly shown that the filament is linearly unstable, with a maximum growth rate of approximately $8.9 \%$ of its internal shear. The preferred mode of instability has a wavelength of approximately 4.3 times the width of the filament. In its nonlinear stages, the instability results in the formation of a series of billows. The billows are connected by weak braids (with lower amplitude buoyancy) which subsequently destabilise at much smaller scales. As such, this instability provides a rapid route to the generation of small scales at the surface. The cascade in physical space is associated with the formation of a shallow $k_{h}^{-5 / 3}$ inertial range in the buoyancy variance spectra. Since the buoyancy variance is equivalent to the surface energy, the $k_{h}^{-5 / 3}$ spectrum indicates a strong forward cascade of energy to small scales.

Additionally, we have shown that the surface buoyancy may, under certain conditions, strongly disrupt an internal vortex. This happens when the vortex is near the surface and the buoyancy anomaly is strong. The form of the interaction depends crucially on the relative directions of the shear associated with the vortex and the filament. In adverse shear, the interaction tends to be significantly more destructive. In cooperative shear, the vortex can largely withstand the strong shear induced by the filament, since that shear tends to make the vortex rotate faster and thus spend less time aligned with the extensional axis of strain.

By analysing the energy components of the interaction, we have found that the self energy of the vortex typically diminishes as it is deformed from its initially spherical shape (in verticallystretched QG coordinates). The greater the deformation, the greater is the loss in vortex self energy. This energy is transferred to the filament-vortex interaction energy and the self energy of the filament. As the filament rolls up into billows, and the connecting braids roll up forming progressively finer scales, the self energy of the filament eventually exhibits a moderate decay (for fixed spatial resolution). This is due to the forward cascade of buoyancy variance, inevitably resulting in dissipation of energy.

We have further explored the effect of a horizontal offset between the centre of the vortex 
and the centre of the filament. For large offsets, the vortex is hardly disturbed but seeds a disturbance on the buoyancy filament which causes it to roll up. For moderate offsets, the vortex is initially propelled along the filament, disturbing different parts as it moves. This leads to a more complex asymmetric interaction.

We have identified a condition for vortex extension in the case of adverse shear, namely that the local horizontal shear at the vortex centre $\partial u / \partial y$ exceeds $0.0768 q$ where $q$ is the PV within the vortex. The shear $\partial u / \partial y$ can be calculated semi-analytically from the initial buoyancy distribution. This condition is based on the behaviour of an initially spherical QG vortex subject to uniform horizontal shear (McKiver and Dritschel (2003)).

As regards oceanographic applications, this idealised study shows that the horizontal location of a surface buoyancy anomaly does not necessarily correspond to a deep vortex below it. In fact, in some cases, a vortex may correspond to a zone depleted of buoyancy. Consequently, deep vortex detection from sea surface temperature (or sea surface density anomaly) alone is generally not reliable, see Carton et al. (2010) and l'Hégaret et al. (2014).

In this study, we have emphasised the buoyancy filament as the archetype flow structure capable of efficiently generating small scales, either on its own or in conjunction with a deep vortex. One can envision other forms of interaction, e.g. with a moving internal structure such a heton or a baroclinic dipole crossing under a buoyancy filament, or with bottom topography. Such studies may provide further insight into some of the fundamental dynamical mechanisms at play in the interaction of surface jets and deeper mesoscale structures, both key ingredients in the oceans circulation.

\section{Appendix 1: derivation of the dispersion relation for a buoyancy filament}

We consider a basic-state buoyancy filament parallel to and centred on the $x$-axis. Starting from the kinematic condition

$$
\frac{D \eta}{D t}=v
$$

which expresses the fact the buoyancy contours are material, $v$ can be calculated in spectral space from

$$
\hat{v}=-\frac{i k}{|\mathbf{k}|} \hat{b}
$$

which uses the inversion relation $\hat{\psi}=-\hat{b} /|\mathbf{k}|$ and $\hat{v}=i k \hat{\psi}$, where $\mathbf{k}=(k, l)$ is the wave vector. Here, $\hat{\xi}$ is the Fourier transform of any variable $\xi$ in physical space.

Since the basic state is independent of $x$ and $t$, we can seek solutions proportional to $e^{i(k x-\sigma t)}$, where $\sigma$ is determined from an eigenvalue problem below. For any physical variable $\xi$, we take

$$
\xi(x, y, t)=-\check{\xi}(y) e^{i(k x-\sigma t)} .
$$

Now since the buoyancy perturbation satisfies $b^{\prime}=-\eta \bar{b}_{y}$ generally, it follows that the same is true for the spectral amplitudes:

$$
\check{b}^{\prime}=-\check{\eta} \bar{b}_{y}=\check{\eta} \frac{b_{m}}{a^{2}} \frac{y}{\sqrt{1-(y / a)^{2}}} .
$$

To obtain $\check{v}$ we carry out, formally, the inverse Fourier transform of $\hat{v}$ with respect to $l$,

$$
\check{v}=\frac{1}{2 \pi} \int_{-\infty}^{\infty} \hat{v} e^{i l y} d l=-\frac{i k}{2 \pi} \int_{-\infty}^{\infty} \frac{\hat{b}^{\prime} e^{i l y}}{\sqrt{k^{2}+l^{2}}} d l,
$$


using (13). Next, to obtain $\hat{b}^{\prime}$ as function of the perturbation amplitude $\check{\eta}$ we carry out a Fourier transform of $\check{b}^{\prime}$ in $y$ :

$$
\hat{b}^{\prime}=\int_{-\infty}^{\infty} \check{b}^{\prime} e^{-i l y^{\prime}} d y^{\prime}
$$

Only $\check{b}^{\prime}$ contributes to $v$ as, in equilibrium, $v=0$. Moreover, because we consider only disturbances which displace the material contours within the filament, then $\check{b^{\prime}} \neq 0$ within the filament only (i.e. for $|y|<a$ after linearisation). Hence, we can rewrite the above and make use of (15) to obtain

$$
\hat{b}^{\prime}=\int_{-a}^{a} \check{b}^{\prime} e^{-i l y^{\prime}} d y^{\prime}=-\frac{b_{m}}{a^{2}} \int_{-a}^{a} \check{\eta} \frac{y^{\prime}}{\sqrt{1-\left(y^{\prime} / a\right)^{2}}} e^{-i l y^{\prime}} d y^{\prime} .
$$

Therefore, using this in (16), we find

$$
\check{v}^{\prime}=\frac{i k b_{m}}{2 \pi a^{2}} \int_{-a}^{a} \check{\eta} \int_{-\infty}^{\infty} \frac{e^{i l\left(y-y^{\prime}\right)}}{\sqrt{k^{2}+l^{2}}} d l d y^{\prime}
$$

where the order of integration has been swapped. For the inner integral, the imaginary part is zero by symmetry while the real part gives

$$
\int_{-\infty}^{\infty} \frac{\cos \left(l\left(y-y^{\prime}\right)\right)}{\sqrt{k^{2}+l^{2}}} d l=2 K_{0}\left(k\left(y-y^{\prime}\right)\right)
$$

where $K_{0}$ is the modified Bessel function of the second kind (from Abramowitz and Stegun, 1964, p 376, formula 9.6.21). The kinematic relation written for $\check{\eta}$ therefore reads

$$
i\left(k U_{e}-\sigma\right) \check{\eta}(y)=\frac{i k b_{m}}{\pi a^{2}} \int_{-a}^{a} \check{\eta}\left(y^{\prime}\right) \frac{y^{\prime}}{\sqrt{1-\left(y^{\prime} / a\right)^{2}}} K_{0}\left(k\left(y-y^{\prime}\right)\right) d y^{\prime},
$$

where $U_{e}=b_{m} y / a$ is the linear shear flow within the filament. The apparent singularity of the integrand at $y^{\prime}= \pm a$ can be removed by the substitution $y^{\prime}=-a \cos \theta^{\prime}$, with $0<\theta^{\prime}<\pi$, for which

$$
\frac{d y^{\prime}}{a \sqrt{1-\left(y^{\prime} / a\right)^{2}}}=d \theta^{\prime}
$$

leading to the final expression

$$
\left(k U_{e}-\sigma\right) \check{\eta}(\theta)=-\frac{i k b_{m}}{\pi} \int_{0}^{\pi} \check{\eta}\left(\theta^{\prime}\right) \cos \theta^{\prime} K_{0}\left(k a\left(\cos \theta^{\prime}-\cos \theta\right)\right) d \theta^{\prime} .
$$

\section{Appendix 2: Sub-surface influence of the buoyancy filament}

In this appendix, we examine how the energy associated with a buoyancy filament is distributed with depth. For this we diagnose the quantity $\hat{b}_{l}^{2}(z)$, the depth dependent horizontal spectrum of $b=\partial \psi_{s} / \partial z$ as well as the spatial distribution of $b^{2}(y, z)$, the potential energy density. Note: the filament is independent of $x$, so $l$ here refers to the $y$ wavenumber.

Starting from the analytical profile of buoyancy at the surface $b(y, z=0)=b_{m} \sqrt{1-(y / a)^{2}}$, we perform a Fourier transform in $y$ to obtain its spectral coefficients $\hat{b}_{l}(z=0)$ and hence the spectral streamfunction $\hat{\psi}_{l}(z=0)=-\hat{b}_{l}(z=0) /|l|$. The depth-dependent streamfunction can then be found by solving

$$
\Delta \psi=\frac{\partial^{2} \psi}{\partial y^{2}}+\frac{\partial^{2} \psi}{\partial z^{2}}=0
$$



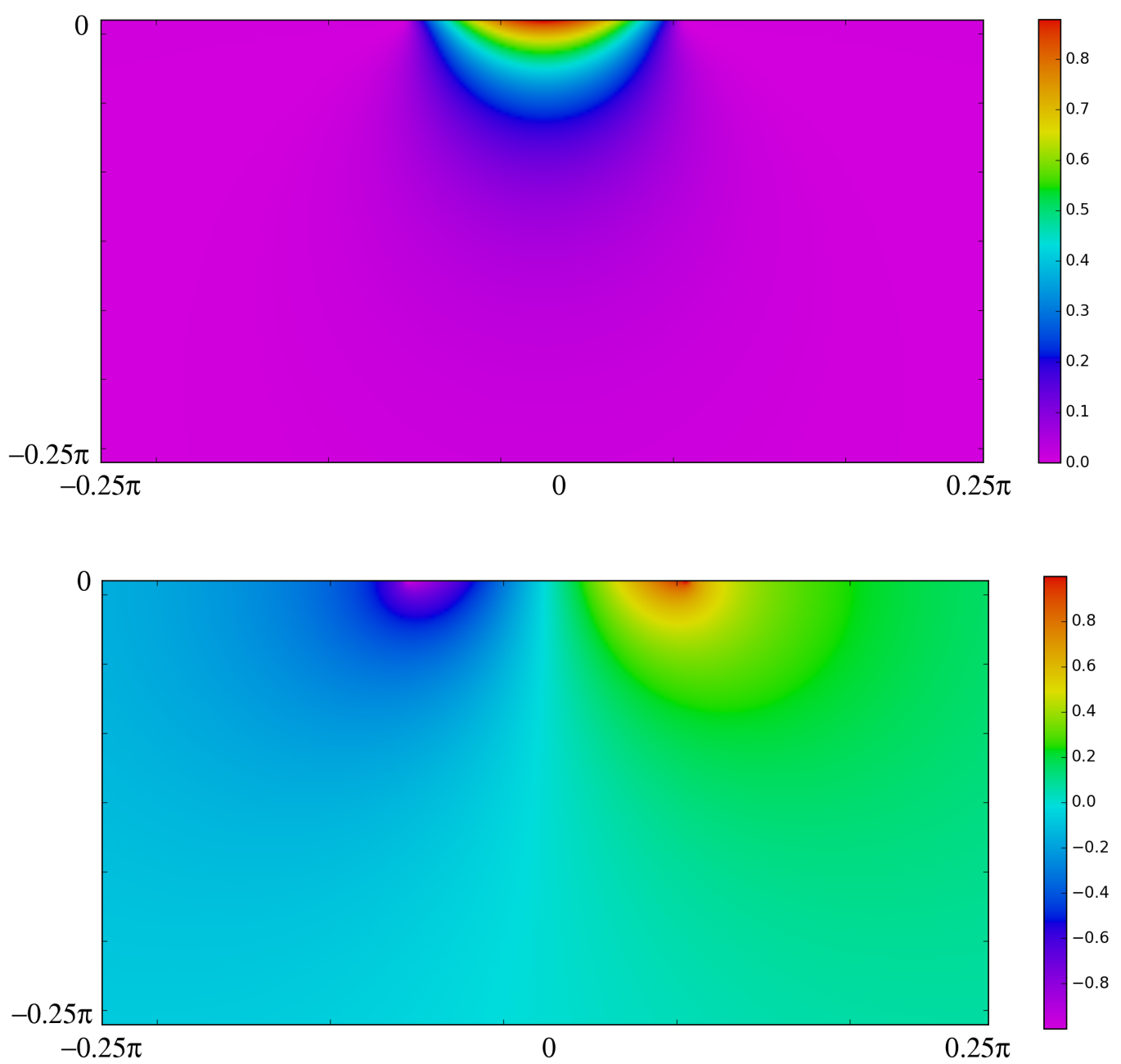

Figure 17. Top: close-up of the spatial distribution of the interior buoyancy squared (potential energy density)) $b^{2}(y, z)$ due to an elliptical buoyancy filament at the upper surface. The domain shown is $-\pi / 4 \leq y \leq \pi / 4$ and $-\pi / 4 \leq z<0$. Bottom: $x$ velocity component $u(y, z)$ in the same domain.

subject to the boundary conditions

$$
\frac{\partial \psi}{\partial z}(y, 0)=b(y, 0), \quad \frac{\partial \psi}{\partial z}(y,-2 \pi)=0 .
$$

After the Fourier transform in $y$, the solution is seen to be

$$
\hat{\psi}_{l}(z)=\frac{\hat{b}_{l}(z=0)}{l \sinh (2 \pi l)} \cosh ((2 \pi+z) l), \quad-2 \pi<z<0,
$$

(see Perrot et al, 2010), from which we determine

$$
\hat{b}_{l}(z)=\hat{b}_{l}(z=0) \frac{\sinh ((2 \pi+z) l)}{\sinh (2 \pi l)}=\hat{b}_{l}(z=0) \frac{e^{l z}-e^{-l(4 \pi+z)}}{1-e^{-4 \pi l}}
$$

by differentiation with respect to $z$.

These spectral coefficients can be used to generate the buoyancy variance spectrum as a function of height, and to reconstruct the spatial field of $b(y, z)$. Here, this is done on a $1024^{2}$ grid. Results are presented in figure 17 for $b_{m}=1$. The top panel shows that almost all the energy is concentrated in the vicinity of the filament, and decays very rapidly away from it, 


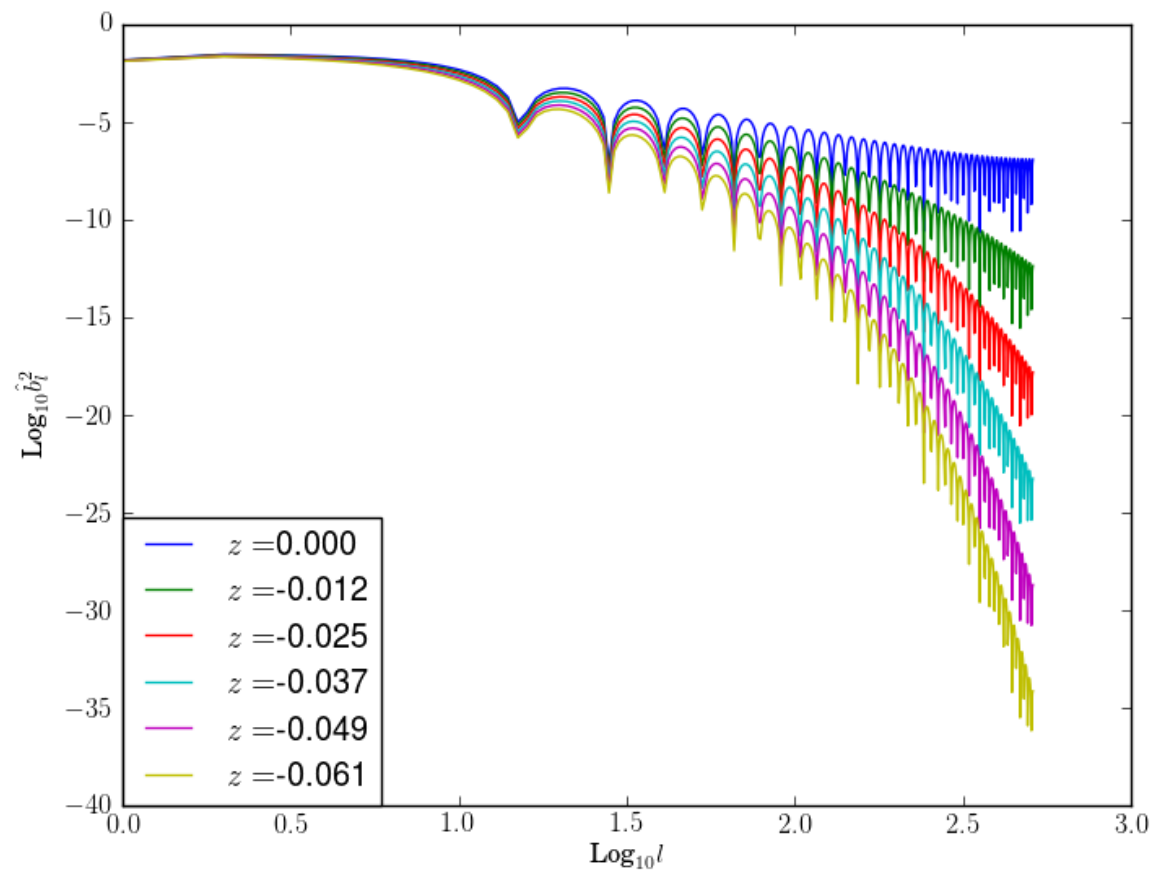

Figure 18. Horizontal spectra of the potential energy $\hat{b}_{l}^{2}$ for different depths. The depth is indicated by layer indices on a $1024^{2}$ grid. The surface is at $z=0$. The bottom is at $z=-2 \pi$, where $b=0$ is imposed.

both in the vertical and horizontal directions. The bottom panel illustrates the structure of the velocity field associated with the buoyancy filament. The peak velocities are located at the edges of the surface filament. The velocity decays rapidly with depth, as does $b$. Figure 18 shows the horizontal spectra of buoyancy variance in the 6 layers nearest the upper surface. Recall that the total depth is $2 \pi$, and the domain is divided into 1024 equal layers. The depth of layer 1014 is therefore only at a depth of less $1 \%$ of the domain depth. Hence, the energy contained at high wavenumbers has a negligible influence on the interior, except for the uppermost few layers.

\section{References}

Barbosa Aguiar, A.C., Peliz, Á., Carton, X., A census of meddies in a long-term high-resolution simulation. Prog. Oceanogr., 2013, 116, 80-94.

Capet, X., Klein, P., Hua, B.L, Lapeyre, G., McWilliams, J.C., Surface kinetic energy transfer in surface quasi-geostrophic flows. J. Fluid Mech., 2009, 604, 165-174.

Carton, X., Daniault, N., Alves, J., Chérubin, L., Ambar, I., Meddy dynamics and interaction with neighbouring eddies southwest of Portugal: Observations and modeling. J. Geophys. Res., 2010, 115, C06017.

Ciani, D., Carton, X., Bashmachnikov, I., Chapron, B., Perrot, X., Influence of deep vortices on the ocean surface. Discontinuity, Nonlinearity, Complexity, 2015, 4(3), 281-311.

Dritschel, D.G., On the stabilization of a two-dimensional vortex filament by adverse shear. J. Fluid Mech., 1989, 206, 193-221.

Dritschel, D.G., The stability of elliptical vortices in an external straining flow. J. Fluid Mech., 1990, 210, $223-261$.

Dritschel, D.G., Haynes, P.H., Juckes, M.N., Shepherd, T.G., The stability of a two-dimensional vorticity filament under uniform strain. J. Fluid Mech., 1991, 230, 647-665.

Dritschel, D.G., Ambaum, M.H.P., A contour-advective semi-lagrangian numerical algorithm for simulating fine-scale conservative dynamical fields. Q. J. Meteorol. Soc., 1997, 123, 1097-1130. 
Dritschel, D.G., Reinaud, J.N., McKiver, W.J., The quasi-geostrophic ellipsoidal vortex model. J. Fluid Mech., 2004, 505, 201-223.

Dritschel, D.G., Fontane, J., The combined Lagrangian advection method. J. Comput. Phys., 2011, 229, 54085417.

Dritschel, D.G., An exact steadily rotating surface quasi-geostrophic elliptical vortex. Geophys. and Astrophys. Fluid Dyn., 2011, 105, 368-376.

Dritschel, D.G., Tobias, S.M., Two-dimensional magnetohydrodynamic turbulence in the small Prandtl Number limit. J. Fluid Mech., 2012, 703, 85-98.

Filyushkin, B.N., Sokolovskiy, M.A., Kozhelupova, N.G., Vagina, I.M., Dynamics of intrathermocline lenses. Doklady Earth Sci., 2010, 434, 1377-1380.

Fontane, J., Dritschel, D.G., The HyperCASL Algorithm: a new approach to the numerical simulation of geophysical flows. J. Comput. Phys., 2009, 228, 6411-6425.

Gula, J., Molemaker, M.J., McWilliams, J.C., Submesoscale Cold Filaments in the Gulf Stream. J. Phys. Ocean., 2014, 44, 2617-2643.

Harvey, B.J., Ambaum, M.H.P., Instability of surface-temperature filaments in strain and shear. Q.J.R. Meteorol. Soc. Part B., 2010, 136, 1506-1513.

Held, I.A., Pierrehumbert, R.T., Garner, S.T., Swanson, K.L., Surface quasi-geostrophic dynamics. J. Fluid Mech., 1995, 282, 1-20.

Iermano, I., Liguori, G., Iudione, D., Buongiorno Nardelli, B., Collela, S., Zingone, A., Saggioma, V., Ribera d'Alcalà, M., Filament formation and evolution in buoyant coastal waters: Observation and modelling. Prog. Oceanogr., 2012, 106, 118-137.

Isern-Fontanet, J., Lapeyre, G., Klein, P., Chapron, B., Hecht, M.W., Three-dimensional reconstruction of oceanic mesoscale currents from surface information. J. Geophys. Res., 2008, 113, C09005.

Juckes, M., Instability of Surface and Upper-Tropospheric Shear Lines. J. Atmos. Sci., 1995, 52, 3247-3262.

Kida, S., Motion of an elliptic vortex in a uniform shear flow. J. Phys. Soc. Jpn., 1981, 50, 3517

Lapeyre, G., Klein, P., Dynamics of the upper oceanic layer in terms of surface quasigeostrophy theory. $J$. Phys. Ocean., 2006, 36, 165-176.

Legras, B., Dritschel, D.G., Vortex stripping and the generation of high vorticity gradients in two-dimensional flows. Appl. Sci. Res., 1993, 51, 445.

L'Hégaret, P. Carton, X., Ambar, I., Ménesguen, C., Hua, B.L., Chérubin, L., Aguiar, A., Le Cann, B., Daniault, N., Serra, N., Evidence of Mediterranean water dipole collision in the Gulf of Cadiz. J. Geophys. Res. Oceans, $2014, \mathbf{1 1 9}, 5337-5359$.

McKiver, W.J,, Dritschel, D.G., The motion of a fluid ellipsoid in a general uniform background flow. J. Fluid Mech., 2003, 474, 147-173.

McWilliams, J.C., Gula, J., Molemaker, M.J., Renault, L., Schchepetkin, A., Filament Frontogenesis by Boundary Layer Turbulence. J. Phys. Ocean., 2014, 45, 1988-2005.

Perrot, X., Reinaud, J.N., Carton, X., Dritschel, D.G., Homostrophic Vortex Interaction under External Strain in a Coupled QG-SQG Model. Regul. Chaotic Dyn., 2010, 15, 66-83.

Richardson, P.L., McCartney, M.S., Maillard, C., A search for meddies in historical data. Dyn. Atmos. Oceans, 1991, 15, 241-265.

Richardson, P.L., Bower, A.S., Zenk, W., Census of meddies tracked by floats. Prog. Oceanogr., 2000, 45, 209-250.

Scott, R.K., A scenario for finite-time singularity in the quasigeostrophic model. J. Fluid Mech., 2011, 687, 492-502.

Scott, R.K., Dritschel, D.G., Numerical simulation of a self-similar cascade of filament instabilities in the surface quasigeostrophic system. Phys. Rev. Lett., 2014, 112, 144505.

Sokolovskiy, M.A., Filyushkin, B.N., Carton, X., Dynamics of intrathermocline vortices in a fire flow over a seamount chain. Ocean Dyn., 2013, 63, 741-760.

Tsang, Y.-K., Dritschel, D.G., Ellipsoidal vortices in rotating stratified fluids: beyond the quasi-geostrophic approximation. J. Fluid Mech., 2015, 762, 196-231.

Tran, C.V., Blackbourn, L.A.K., Scott, R.K., Number of degrees of freedom and energy spectrum of quasigeostrophic turbulence. J. Fluid Mech., 2011, 684, 427-440.

Trieling, R.R., Dam, C.E.C., van Heijst, G.J.F., Dynamics of two identical vortices in linear shear. Phys. Fluids, 2010, 22, 117104.

Vallis, G.K., Atmospheric and Oceanic Fluid Dynamics: Fundamentals and Large-scale Circulation, C.U.P., 2006.

Wang, J., Flierl, G.R., LaCasce, J.H., McLean, J.L., Mahadevan, M., Reconstructing the Ocean's Interior from Surface Data. J. Phys. Ocean., 2013, 43, 1611-1626. 